\title{
Thoracic Low-dose CT Image Processing Using an Artifact Suppressed Large-scale Nonlocal Means
}

\author{
Yang Chen ${ }^{1,2,3,4}$, Zhou Yang ${ }^{1,2}$, Yining $\mathrm{Hu}^{1,2}$, Guanyu Yang ${ }^{1,2}$, Yongcheng Zhu ${ }^{1,2}$, Yinsheng Li $^{1,2}$, \\ Limin luo ${ }^{1,2}$, Wufan Chen ${ }^{5}$, Christine Toumoulin ${ }^{2,3,4}$ \\ 1. Laboratory of Image Science and Technology, Southeast University, Nanjing, China; \\ 2. Centre de Recherche en Information Biomedicale Sino-Francais (LIA CRIBs), F-35042 Rennes, France; \\ 3. Laboratoire Traitement du Signal et de l'Image (LTSI), Université de Rennes, F-35042 Rennes, France; \\ 4. INSERM - U 1099, Université Rennes, F-35042 Rennes, France \\ 5. School of Biomedical Engineering, Southern Medical University, Guangzhou, 510515, China.
}

\begin{abstract}
The x-ray exposure to patients has become a major concern in Computed Tomography (CT) and minimizing the radiation exposure has been one of the major efforts in CT field. Due to the plenty high-attenuation tissues in human chest, under low dose scan protocols, thoracic low-dose CT (LDCT) images tend to be severely degraded by excessive mottled noise and non-stationary streak artifacts. Their removal is rather a challenging task because the streak artifacts with directional prominence are often hard to be well discriminated from the attenuation information of normal tissues. This paper describes a two-step processing scheme called "Artifact Suppressed Large-scale Nonlocal Means" (AS-LNLM) for suppressing both noise and artifacts in thoracic LDCT images. Specific scale and direction properties were exploited to discriminate the noise and artifacts from image structures. Parallel implementation has been introduced to speed up the whole processing by more than 100 times. Phantom and patient CT images were both acquired for evaluation purpose. Comparative qualitative and quantitative analyses were both performed that allows concluding on the efficacy of our method in improving thoracic LDCT data.
\end{abstract}

Key Words: Artifact Suppressed Large-scale Nonlocal Means (AS-LNLM), Low-dose CT (LDCT), Stationary wavelet transform (SWT), Streak artifacts.

\section{Introduction}

The lung and bronchus cancer is a leading cause of cancer death in the United States with a mortality rate that amounts to $29 \%$ [1]. Computed tomography (CT) is widely used for lung cancer screening because it is more sensitive than chest radiography in the detection of small nodules and lung carcinoma at an early stage [2-3]. Nevertheless, a trouble spot is the x-ray dose delivered by the scanner. It is indeed given to be relatively high $(5-15 \mathrm{mSv})$, which may be dangerous at more or less long term for patients having to pass several CT examinations over a certain period of time. Thus to limit the radiation dose, low-dose computed tomography (LDCT) could be applied for screening in patients at high risk for developing lung cancer [4]. Low dose CT can be achieved by decreasing the milliamperage and the voltage [5-6], which, however, leads to a degraded signal to noise ratio [7-11]. This is due to a severe increase of the quantum and electronic noise. As a consequence, reconstructed images appear degraded by the presence of mottled noise and pronounced streak artifacts [4-8]. It might be difficult, thus for radiologists to distinguish between benign and malignant nodules on LDCT. A recent report of the American Society of Clinical Oncology emphasizes that the number of false-positive in the detection of lung nodules, was high for individuals who underwent lung cancer screening with low-dose computed tomography (LDCT): $21 \%$ after a first LDCT scan, and 33\% after a second one [7]. 
The streak artifacts, taking the appearance of directional patterns, are often prominent in thoracic LDCT images and on structures that have extremely high attenuation. Their suppression is rather challenging due to their orientation prominences, which are often similar to those of structures that have normal attenuation. Many methods have been proposed to improve the quality of thoracic LDCT images, which can be roughly divided into two categories: Raw data-based and post-processing techniques.

The first one refers to raw data-based techniques which directly locate in the projection space. Raw data-based techniques improve the LDCT reconstruction by restoring the projected raw data or iterative solving a prior-regularized energy function [8-13]. Researches in this direction are however always limited by the difficulty to access the well-formatted CT projection data and the high involved computation cost [8-13].

Post-processing techniques are applied directly on the reconstructed image data but because the mottled noise and non-stationary artifacts in LDCT images can not be well modeled into a generic distribution, it is often difficult to well differentiate between noise/artifacts and anatomical/pathological data [14-20]. Different techniques have been proposed for improving the quality of LDCT images. For instance, a hybrid approach, making use of low-pass and directional filters for segmented both non-structured and structured regions, has been described in [14]. In [15], a filter named SharpView CT applies a multi frequency analysis to divide the image into several sub-bands and separately process them before re-combination. In [16], a feature-dependent operation was used to reduce noise and streak artifacts around the thoracic inlet by applying different processing on different structures. A diffusion filter modulated by local neighboring information, was applied in [17] and [18] to process thoracic LDCT images. In [19], a Hybrid anisotropic Diffusion filter with a Continuous Switch (HDCS) exploited local eigenvalue information to determine the local feature shape and apply a differentiated enhancing diffusion process. In [20], a weighted intensity averaging over large-scale neighborhoods (WIA-LN) was proposed for processing abdominal LDCT images, which can be deemed as a large-scale nonlocal means (LNLM) [21-23]. The weighted large-scale averaging in the LNLM method relies on the information redundancy property within a local neighborhood to suppress mottled noise without obvious loss of image details. Nevertheless, as also pointed out in [20], the LNLM method is not effective in suppressing the non-stationary streak artifacts in thoracic CT images.

This paper describes an Artifact Suppressed Large-scale Non Local Means (AS-LNLM) method to process thoracic LDCT images. It exploits scale and directional properties for suppressing noise and artifacts and relies on a two stage processing scheme. The first one aims at suppressing streak artifacts in the LDCT images by applying a directional 1-D nonlinear diffusion in the stationary wavelet domain. The second stage makes then use of a LNLM filtering for denoising the artifact suppressed images (Section II). To reduce the computational complexity, a multithreading implementation has been carried out that take advantage of the Compute Unified Device Architecture (CUDA) [24-27]. In sections III and IV, experiments were both conducted on a 2D thoracic anthropomorphic phantom and real data acquired from a Siemens 16 detector rows CT. A first qualitative comparison with the latest iterative algorithm developed by GE company is presented. A qualitative and quantitative study is then performed on the thoracic phantom and a comparison with the HDCS filter [19] is provided. Results are lastly given on real data with an expert-based evaluation, which shows the proposed method achieved effective noise/artifact reduction in thoracic LDCT images with little compromise in contrast.

The list of abbreviations below will be used thereafter:
LDCT
Low-dose CT
HDCT
HDCS
High-dose CT
LNLM
AS-LNLM
Hybrid Diffusion filter with a Continuous Switch in [19]
CUDA
Large-scale Nonlocal Means in [20]
Artifact Suppressed Large-scale Nonlocal Means
Compute Unified Device Architecture 


\section{Methods}

\section{A. The Original LNLM Method}

The Non Local Means (NLM) Filter, originally introduced by Buades et al. [21] for 2D image denoising, relies on the information redundancy within a neighbourhood. The basic idea is to replace the value of a pixel by the weighted average of pixels located in a search neighborhood window of size $N$. Each weight expresses the similarity in intensity between the central pixel in the window and each neighboring pixel and is given by the pair-wise difference between patches surrounding each pair of considered pixels. Some adaptations have been proposed for optimizing the estimation of this pixel considering the selection of the most relevant pixels in the search neighborhood [22], the block-wise computation [23] or a large scale neighborhood [20].

We consider here our adaptation of the NLM filter that is Large scale Non-Linear Means Filter (LNLM) [20]. Let $f_{i}$ and $\hat{f}_{i}$ denoting the intensities of pixel $i$ before and after processing, the LNLM method can be outlined as follows:

$$
\begin{gathered}
\hat{f}_{i}=\sum_{j \in N_{i}} w_{i j} f_{j} / \sum_{j \in N_{i}} w_{i j} \\
w_{i j}=\exp \left(-\left\|v\left(n_{i}\right)-v\left(n_{j}\right)\right\|_{2, \alpha}^{2} / h|n|\right), \quad v\left(n_{i}\right)=\left\{f_{l}: l \in n_{i}\right\} ; v\left(n_{j}\right)=\left\{f_{l}: l \in n_{j}\right\}
\end{gathered}
$$

where, $N_{i}$ is the search neighborhood centered at pixel $i, j$ the pixels located in the neighborhood of $i$. $w_{i j}$ characterizes the pair-wise similarity measure between the two patches $n_{i}$ and $n_{j}$ surrounding pixel $i$ and $j$, respectively. We define $v\left(n_{i}\right)$ and $v\left(n_{j}\right)$ as the two pixel intensity vectors which include all the pixels in the two patches $n_{i}$ and $n_{j}$, respectively. The $w_{i j}$ in (2) is calculated as the attenuating function of the Gaussian-weighted distance $\left\|v\left(n_{i}\right)-v\left(n_{j}\right)\right\|_{2, \alpha}^{2}$ between the two patches $n_{i}$ and $n_{j}$. $|n|$ denotes the pixel number in $n$, and is used as a normalization parameter to make the processing independent of the different size settings of $n . \alpha$ denotes the standard deviation of the Gaussian kernel. Parameter $h$ is used to control the smoothing effect in the attenuating exponent function in (2). The application of the LNLM method is based on the assumption that in clinical CT images the pixels representing different tissue attenuation often distribute over a large scale, ad pixels of similar surrounding distribution have a higher probability to belong to the same tissues. The main contribution of [20] has been to demonstrate that the weighted intensity averaging within large searching neighborhood (such as $81 \times 81$ or $161 \times 161 \mathrm{~N}$ ) can lead to effective suppression of the mottled noise in low-dose abdominal CT images. It has been shown in [20] that, with up to one fifth of the routine tube current setting, clinically acceptable abdominal LDCT images can be obtained by using this LNLM method.

\section{B. The Proposed AS-LNLM Method}

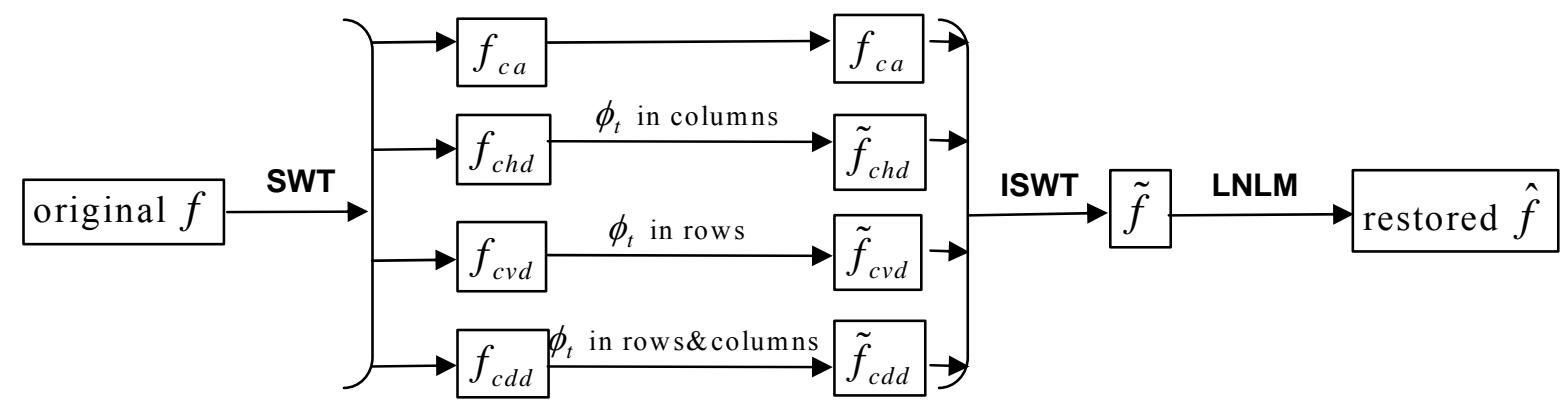

Fig. 1. The outline of the proposed AS-LNLM method. $f$ denotes the original degraded LDCT image. $f_{c a}$ denote all the wavelet decomposed low frequency $2 \mathrm{D}$ subbands. $f_{c h d}, f_{c v d}, f_{c d d}$ and $\tilde{f}_{c h d}, \tilde{f}_{c v d}, \tilde{f}_{c d d}$ respectively denote the 
original and nonlinear diffusion processed high frequency 2D subbands (horizontal, vertical and diagonal). $\phi_{t}$ is the 1-D nonlinear diffusions applied on each extracted 1D column or row data, which are in the orthotropic directions to the high frequency components in the high frequency subbands. $\tilde{f}$ is the reconstructed image from the subbands $\left(f_{c a}, \tilde{f}_{c h d}, \tilde{f}_{c v d}, \tilde{f}_{c d d}\right) . \hat{f}$ is the final processed LDCT image from the LNLM operation of $\tilde{f}$.

It was pointed out in [20] that the LNLM method was not effective in suppressing the streak artifacts in thoracic LDCT images because the directional patterns of streak artifacts often prevent an effective suppression. To overcome this, we devised a two-step processing AS-LNLM that applies different artifact/noise reduction in different scales. In this method (outlined in Fig.1), the streak artifacts in LDCT images are pre-suppressed in wavelet domain by directional 1-D nonlinear diffusions before the LNLM operation. To preserve the position invariance for each decomposed 2D subband, here the translation-invariant Stationary Wavelet Transform (SWT) is used [28]. Compared to the familiar discrete wavelet transform, at each scale in SWT the translation-invariance does not perform downsampling, but otherwise upsamples the filter coefficients by a factor of two [28-29]. The Haar wavelet is used in SWT for it is fast and found to suffer less from the so-called "ringing" or pseudo-Gibbs artifacts when compared to other wavelets with wider filter bases [30-31].

To achieve effective artifact suppression, the 1-D nonlinear diffusions are applied along the directions orthotropic to the high frequency orientations in the decomposed 2D wavelet subbands. For the high frequency subbands of $f_{c h d}$ and $f_{c v d}$ (horizontal and vertical), we perform 1D column-wise and row-wise nonlinear diffusions ((4)-(5)). To suppress artifacts in the diagonal high frequency subbands $f_{c d d}$, we sequentially perform the $1 \mathrm{D}$ column-wise and row-wise nonlinear diffusions through $((6)-(7))$. The whole processing includes the following (3)-(11):

$$
\begin{gathered}
{\left[f_{c a}, f_{c h d}, f_{c v d}, f_{c d d}\right]=\operatorname{SWT}(f, S)} \\
\tilde{f}_{c h d}(:, c o l, s)=\phi_{t}\left(f_{c h d}(:, c o l, s)\right), \text { for } \operatorname{col}=1,2, \ldots . . C, s=1,2, \ldots . . S \\
\tilde{f}_{c v d}(\text { row },:, s)=\phi_{t}\left(f_{c v d}(\text { row, }:, s)\right), \text { for } \operatorname{row}=1,2, \ldots . . R, s=1,2, \ldots . . S \\
\tilde{f}_{c d d}(:, c o l, s)=\phi_{t}\left(f_{c d d}(:, c o l, s)\right), \text { for } c o l=1,2, \ldots . . C, s=1,2, \ldots . . S \\
\tilde{f}_{c d d}(\text { row },:, s)=\phi_{t}\left(f_{c d d}(\text { row },:, s)\right), \text { for } \operatorname{row}=1,2, \ldots . . R, s=1,2, \ldots . . S \\
\phi_{t}(I)=\operatorname{div}(c(\nabla I(t)) \nabla I), c(\nabla I(t))=\exp \left(-(\|\nabla I(t)\| / K)^{2}\right) \\
\tilde{f}=\operatorname{ISWT}\left(\left[f_{c a}, \tilde{f}_{c h d}, \tilde{f}_{c v d}, \tilde{f}_{c d d}\right], S\right) \\
\hat{f}_{i}=\sum_{j \in N_{i}} w_{i j} \tilde{f}_{j} / \sum_{j \in N_{i}} w_{i j} \\
w_{i j}=\exp \left(-\left\|v\left(n_{i}\right)-v\left(n_{j}\right)\right\|_{2, \alpha}^{2} / h|n|\right)
\end{gathered}
$$

In (3), at each scale $s$ in SWT, the original LDCT image $f$ is decomposed into three high frequency 2D subbands $\left[f_{c h d}(:,:, s), f_{c v d}(:,:, s), f_{c d d}(:,:, s)\right]$ and one low frequency $2 \mathrm{D}$ subband $f_{c a}(:,:, s)$. In (4)-(7), $S$ denotes the total decomposition scales, and $C$ and $R$ denote respectively the column and row dimensions for each decomposed 2D subbands. In (8) $\phi_{t}$ represents the 1-D nonlinear diffusions applied on each extracted 1D column or row data $I$ with the scale-space variable $t$ in the diffusion process [30]. Defined as a monotonically decreasing function of the gradient $\nabla I$, function $c$ is set as the divergence function $\exp \left(-(|\nabla I(t)| / K)^{2}\right)$ with $K$ controlling the tradeoff between artifact-suppression and edge-preservation [32]. In (9), with the original low frequency subband $f_{c a}$ and the three processed high 
frequency subbands $\tilde{f}_{c h d}, \tilde{f}_{c v d}$ and $\tilde{f}_{c d d}$, the Inverse Stationary Wavelet Transform (ISWT) is carried out to provide the artifact-suppressed image $\tilde{f}$. The final restored image $\hat{f}$ can be then obtained through the application of LNLM (equations (10)-(11)) of $\tilde{f}$.

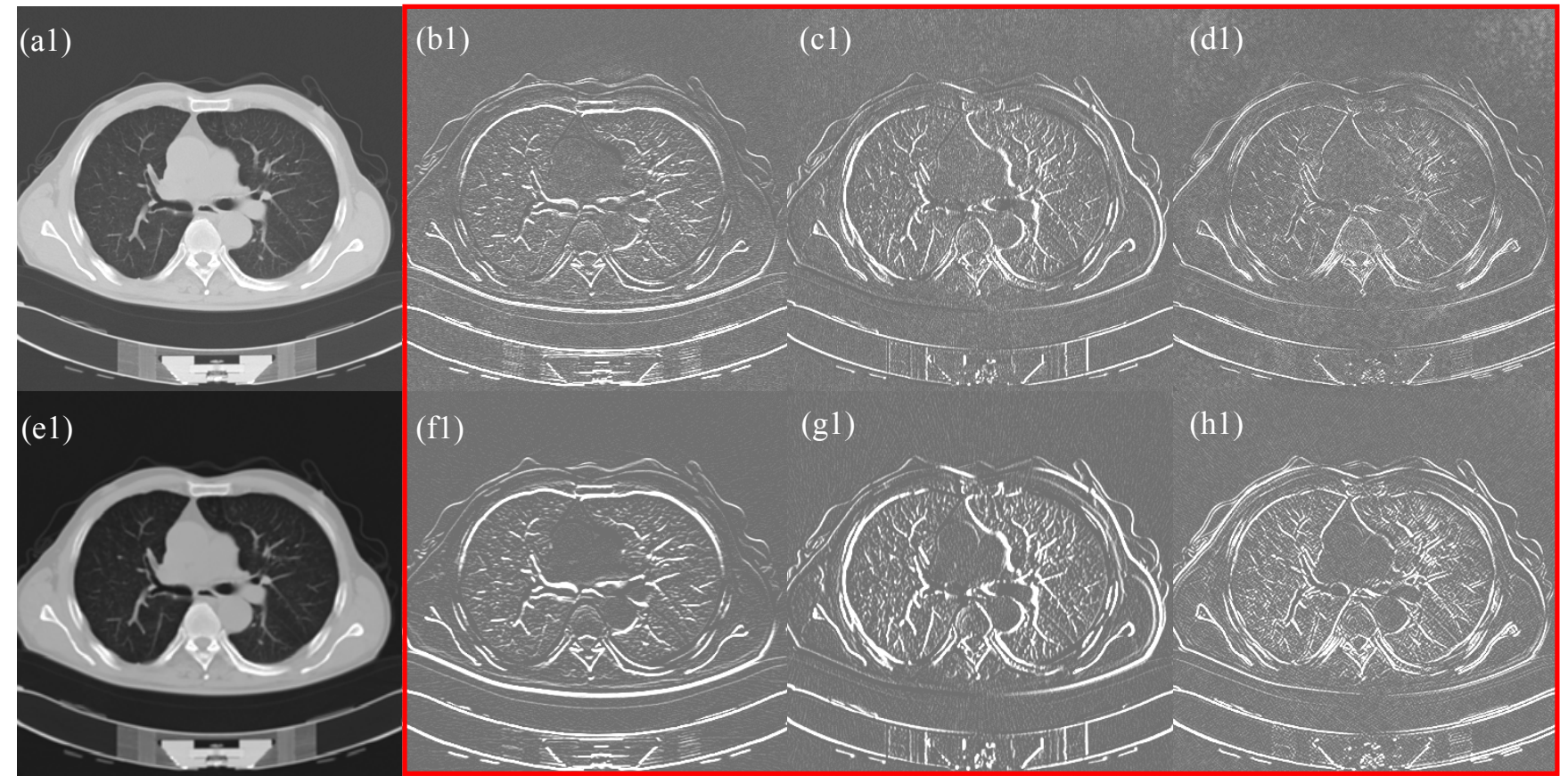

(1), Decomposed subbands of a typical HDCT image (120mAs). (a1)-(d1): for the first scale, the low frequency subband $f_{c a}(:,:, 1)$, the horizontal high frequency subband $f_{\text {chd }}(:,:, 1)$, the vertical high frequency subband $f_{c v d}(:,:, 1)$ and the diagonal high frequency subband $f_{c d d}(:,:, 1)$. (e1)-(h1): for the second scale, the low frequency subband $f_{c a}(:,:, 2)$, the horizontal high frequency subband $f_{c h d}(:,:, 2)$, the vertical high frequency subband $f_{c v d}(:,:, 2)$ and the diagonal high frequency subband $f_{c d d}(:,:, 2)$.

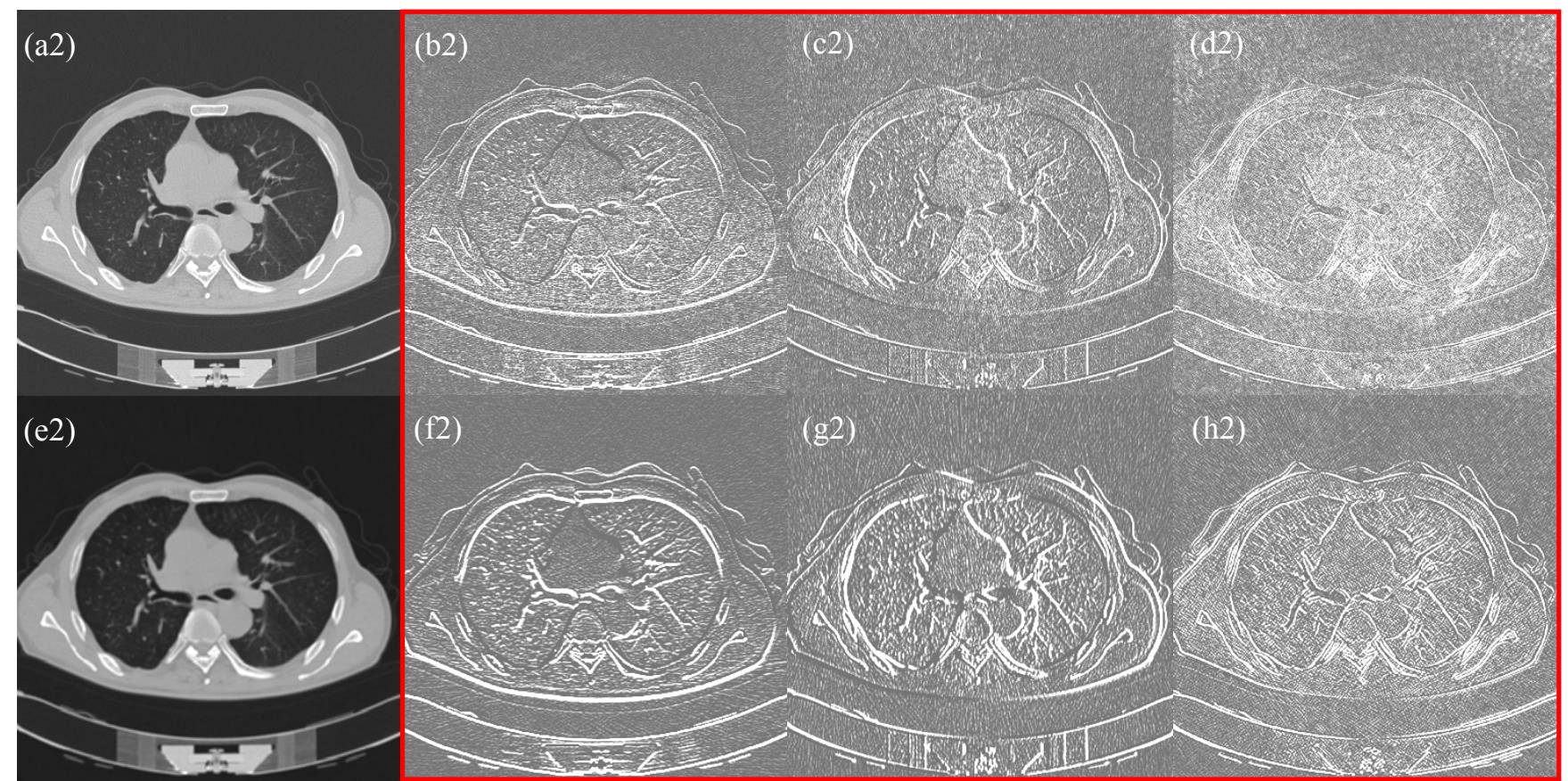

(2), Decomposed subbands of a typical LDCT image (30mAs) with the similar body position as above HDCT images. (a2)-(d2): for the first scale, the low frequency subband $f_{c a}(:,:, 1)$, the horizontal high frequency subband $f_{\text {chd }}(:,:, 1)$, the vertical high frequency subband $f_{c v d}(:,:, 1)$ and the diagonal high frequency subband $f_{c d d}(:,:, 1)$. (e2)-(h2): for 
the second scale, the low frequency subband $f_{c a}(:,:, 2)$, the horizontal high frequency subband $f_{\text {chd }}(:,:, 2)$, the vertical high frequency subband $f_{c v d}(:,:, 2)$ and the diagonal high frequency subband $f_{\mathrm{cdd}}(:,:, 2)$.
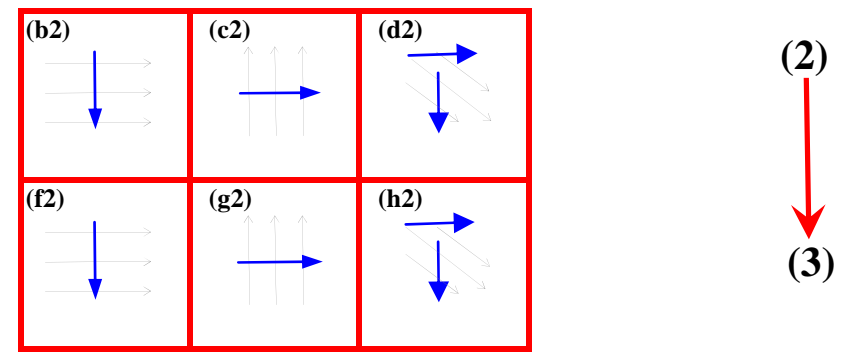

(3)

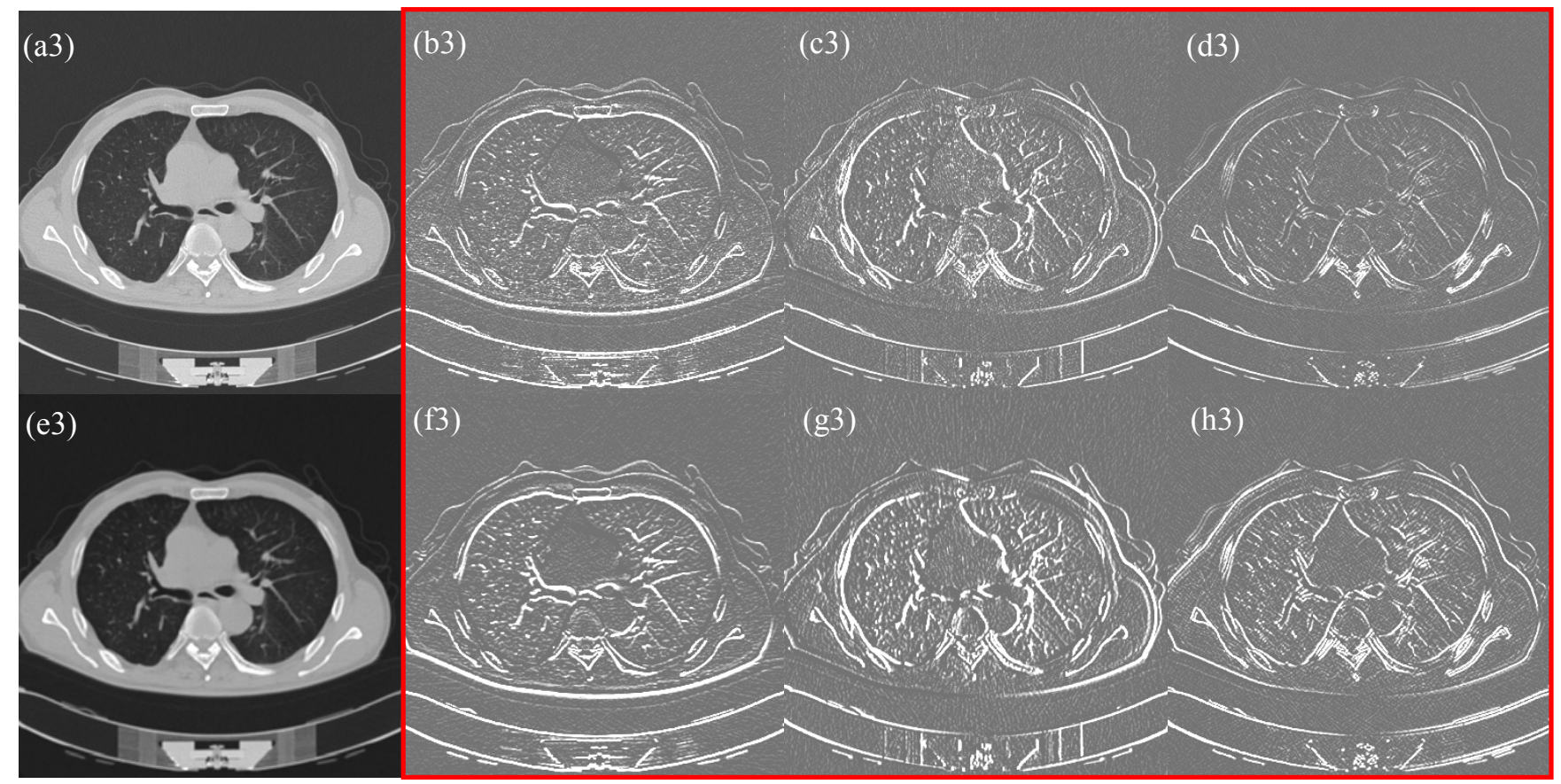

(3), Decomposed subbands of the LDCT image in (2). (a3)-(d3): for the first scale, the original low frequency subband $f_{c a}(:,:, 1)$, the processed horizontal high frequency subband $\tilde{f}_{c h d}(:,:, 1)$, the processed vertical high frequency subband $\tilde{f}_{c v d}(:,:, 1)$ and processed the diagonal high frequency subband $\tilde{f}_{\text {cdd }}(:,:, 1)$. (e3)-(h3): for the second scale, the original low frequency subband $f_{c a}(:,:, 2)$, the processed horizontal high frequency subband $\tilde{f}_{c h d}(:,:, 2)$, the processed vertical high frequency subband $\tilde{f}_{c v d}(:,:, 2)$ and the processed diagonal high frequency subband $\tilde{f}_{c d d}(:,:, 2)$.

Fig. 2. Illustration of the 1D directional nonlinear diffusions in wavelet domain (Haar wavelet, two scales $(S=2)$ ) in the proposed method. Note that this is only for illustration purposes. One typical LDCT and HDCT image are used. In the central table above Fig.2 (3), the blue and grey arrows represent the directions of the applied nonlinear diffusions together with the high frequency components in the decomposed subbands, respectively.

Fig. 2 illustrates the decomposition of the high frequency components for a LDCT image and depicts in the table (between Fig. 2 (2) and (3) the directions for the applied nonlinear diffusions and also the high frequency components in decomposed subbands. The nonlinear diffusions were applied based on (4)-(8) with $K=200, S=2,10$ iterations and an increment equal to 0.05. Fig.2 (1) and (2) display the decomposed wavelet subbands for a thoracic HDCT image and LDCT image, respectively. In Fig.2 (2), we can see that, with respect to the illustration of HDCT image in Fig.2 (1), the streak artifacts in LDCT images are much more prominent (with higher amplitudes) in the decomposed high frequency subbands (Fig.2 (2) (b2)-(d2) and (f2)-(h2)) than in the decomposed low frequency subbands (Fig.2 (2) (a2) and (e2)). It can be observed in Fig.2 (3) that the high frequency artifact components were notably suppressed through the developed wavelet domain directional 1D nonlinear diffusions 


\section{Experiment settings}

Approval of this study was granted by our institutional review board. One anthropomorphic phantom and 25 patients have been involved in the experiments. The study, including the data collection and processing, was conducted according to the authorized protocol. All the 25 patients have given their written consent to the participation and received remuneration for it. A non-conflict of interest for this work was declared. CT images were acquired on a multi-detector row Siemens Somatom Sensation 16 CT scanner.

The thoracic phantom is an anatomical model of a human chest torso (Fig. 3). Its size is $43 \times 40 \times 48 \mathrm{~cm}$ and its measurements are the following: chest size $94 \mathrm{~cm}$, height $45 \mathrm{~cm}$ and weight $18 \mathrm{~kg}$. The inner components consists of mediastinum (heart, trachea), pulmonary vasculature and an abdomen block. The thickness of the chest wall is based on measurements of clinical data, and the soft tissue substitute material and synthetic bones have x-ray absorption rates close to those of human tissues.

The patient cohort includes 9 women and 16 men with an average age of 64 years (age range: 52-71 years). 8 of all the patients have verified lung cancer with lesions in the range $5 \mathrm{~mm}-12 \mathrm{~mm}$.
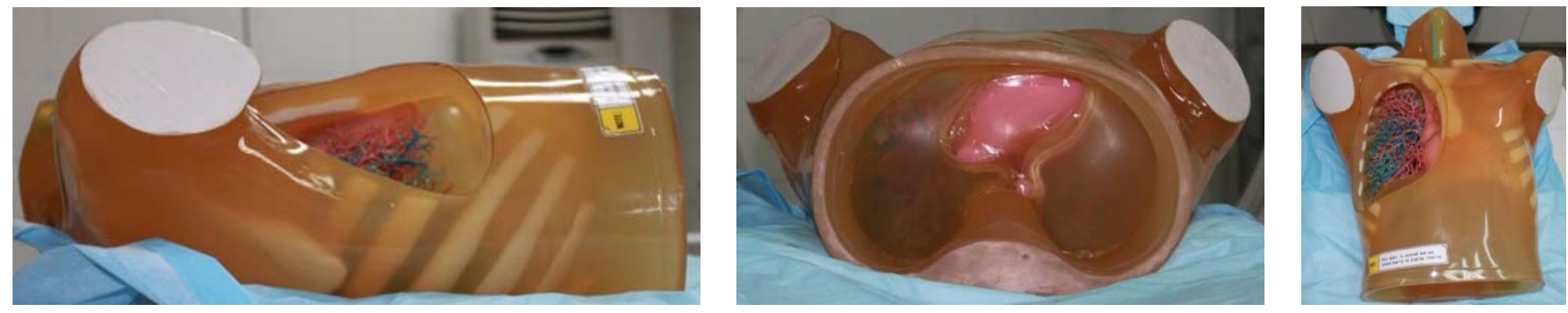

Fig. 3. The anthropopathic thoracic phantom used in experiment.

For both phantom and patient experiments, the LDCT images were collected by using the reduced 30 $\mathrm{mAs}$ (routine clinical mAs setting is $120 \mathrm{mAs}$ ) under chest scanning mode. For each phantom and patient scan, 40 slices were collected. Other scanning parameters were: kVp, 120; Slice thickness, 2 mm; Gantry rotation time, $0.5 \mathrm{~s}$; detector configuration (number of detector rows $\times$ section thickness), $16 \mathrm{~mm} \times 1.5 \mathrm{~mm}$; table feed per gantry rotation, $24 \mathrm{~mm}$; pitch, 1:1; reconstruction method: Filtered Back Projection (FBP) algorithm with convolution kernel "B70f" and "B31f" ("B31f" is the smoothing reconstruction kernel for mediastinal window illustration in Siemens CT). The CT dose index volume $\left(\mathrm{CTDI}_{\mathrm{vol}}\right)$ is a linear function of the tube currents [2]. We recorded the accumulated doses from the workstation for each scan with 40 slices. The recorded doses are $9.36 \mathrm{mGy}$ for the routine (or standard) 120mAs protocol, and $2.34 \mathrm{mGy}$ for the low dose 30mAs protocol. For the phantom experiment, to obtain a high-quality reference volume for quantitative evaluation, HDCT images were acquired with a higher current of $240 \mathrm{mAs}$ because there is no radiation concern for phantom. The experiments aim at illustrating the behavior of the algorithms on chest structures and pulmonary vessels. The windows and level setting are thus chosen to optimize the visualization of these data. We will so consider the mediastinal (window center, 50HU; window width, $350 \mathrm{HU}$ ) and lung (window center, -600HU; window width, 1600HU) windows respectively [33].

For evaluation purposes, we compared the HDCS filter in [19] and the LNLM method described in Section II. We applied the hybrid diffusion filter with a continuous switch (HDCS) method for LDCT images by using the code provided by the author of [19] (http://www.insight-journal.org/browse/publication/748). This HDCS method uses local eigenvalue information to determine whether the local structures are tubular or planar, and then apply either the coherence-enhancing diffusion (CED) or edge-enhancing diffusion (EED) based on the calculated eigenvalue information. All the CT images were exported as DICOM files and then processed offline under a PC workstation (Intel Core ${ }^{\mathrm{TM}} 2$ Quad CPU and 4096 Mb RAM, GPU (NVIDIA GTX465)) with Visual $\mathrm{C}++$ as the developing language (Visual Studio 2008 software; Microsoft).

In the latter LNLM step of the AS-LNLM method, intensive computation is required in the distance 
calculation between each two translated patches $n$ in each neighborhood $N$. In our experiments, we applied a size for the search neighborhood $N$ equal to $81 \times 81$ and for the patches $n 7 \times 7$, which is found practically robust to provide good results. A GPU parallel implementation with CUDA framework has been designed to accelerate the processing. Threads, blocks and grids make up of the physical structure of a CUDA multithreading frame [24-25]. We set the total number of blocks in grid to the row size of the image, and the total number of threads in each block to the column size of the image. In the processing of LNLM, all threads in the block-grid structure execute simultaneously to perform all the pixel-wise operations, which include the pixel-wise 1D nonlinear diffusion in (4)-(8), the pixel-wise weight calculations for each patch pairs in (13), and the pixel-wise $\hat{f}_{i}$ calculations in (10). We also further reduced the computation reduction by applying the parallelization optimization in [26-27]. Runtime comparison in the practical experiments indicates that the parallelized operation is more than 100 times faster than the previous serial version.

\section{Results}

\section{A. Phantom Data Experiment}

TABLE I. THE PARAMETER SETTINGS OF DIFFERENT METHODS IN THE PHANTOM EXPERIMENT ON LDCT IMAGES

\begin{tabular}{|c|c|c|}
\hline & Mediastinum window & Lung window \\
\hline $\begin{array}{c}\text { Reference Images } \\
\text { Processed HDCT images From AS-LNLM) }\end{array}$ & $\begin{array}{c}S=2, K=50, \text { iter }=10, \text { inc }=0.05, \\
h=10,7 \times 7 n, 81 \times 81 N\end{array}$ & $\begin{array}{c}S=2, K=20, \text { iter }=10, \text { inc }=0.05, \\
h=5,7 \times 7 n, 81 \times 81 N\end{array}$ \\
\hline Processed LDCT images from HDCS & $\sigma=1, \lambda_{e}=20, \lambda_{c}=15, \lambda_{h}=20, \alpha=0.001, \tau=0.11, \eta=4$ \\
\hline Processed LDCT images from LNLM (small h) & $h=20,7 \times 7 n, 81 \times 81 N$ & $h=10,7 \times 7 n, 81 \times 81 N$ \\
\hline Processed LDCT images from LNLM (large h) & $h=200,7 \times 7 n, 81 \times 81 N$ & $h=100,7 \times 7 n, 81 \times 81 N$ \\
\hline Processed LDCT images from AS-LNLM & $\begin{array}{c}S=2, K=50, \text { iter }=10, \text { inc }=0.05, \\
h=20,7 \times 7 n, 81 \times 81 N\end{array}$ & $\begin{array}{c}S=2, K=50, \text { iter }=10, \text { inc }=0.05, \\
h=10,7 \times 7 n, 81 \times 81 N\end{array}$ \\
\hline
\end{tabular}
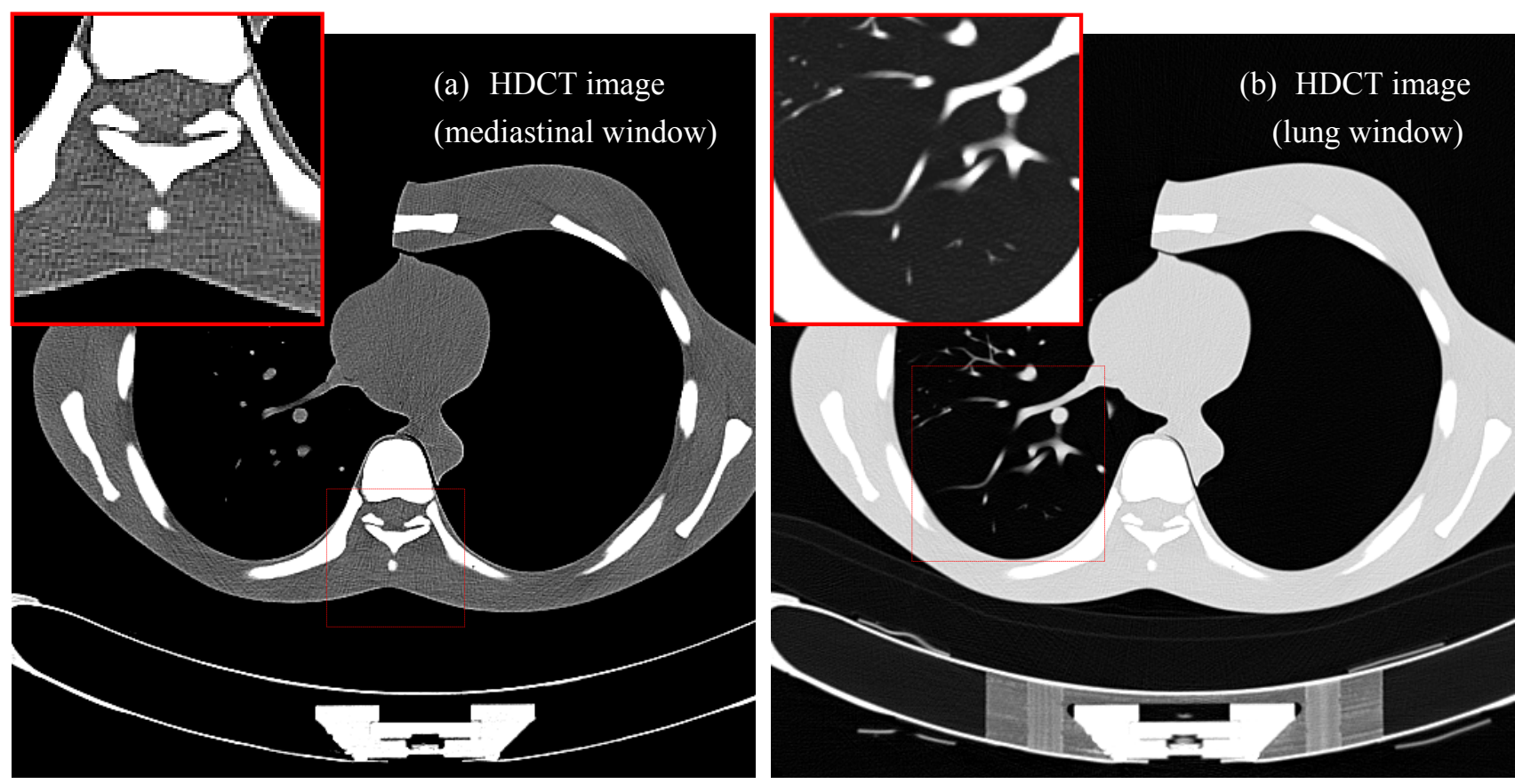

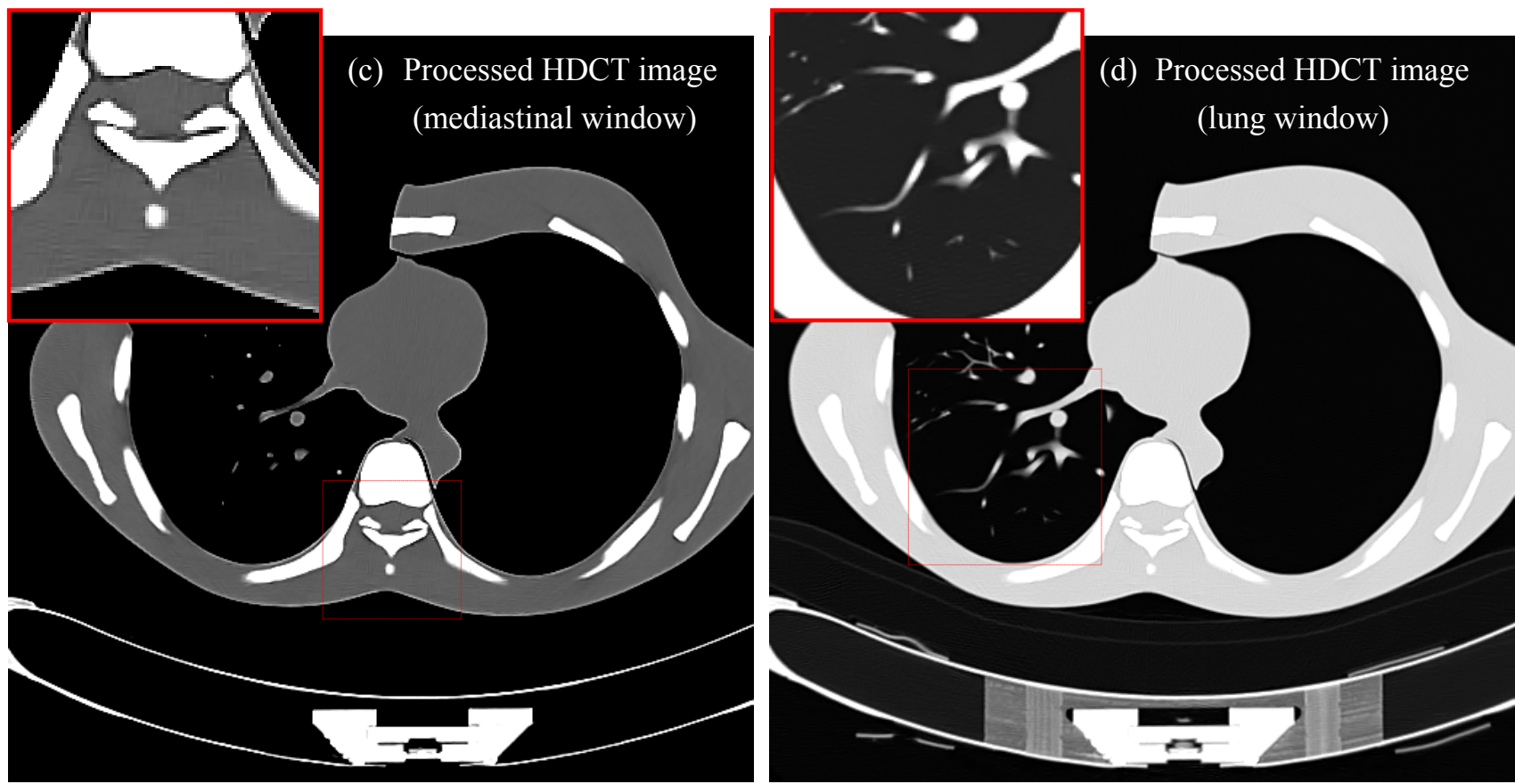

Fig. 4. $16^{\text {th }}$ slice of the volume acquired in HDCT condition for the thoracic phantom. (a) and (b): visualization in mediastinal and lung windows of the original HDCT image; (c) Processed HDCT image from the AS-LNLM method with $[K=50, h=10]$ in mediastinal window; (d), Processed HDCT image from the AS-LNLM method with $[K=20, h=5]$ in lung window

Fig.4 (a) and (b) illustrate one typical HDCT image $\left(240 \mathrm{mAs}\right.$, the $16^{\text {th }}$ slice in the whole data volume) in mediastinal and lung windows. We can see in Fig.4 (a) and (b) that, even with high tube current setting, noise and artifacts still remain in the reconstructed images (c.f. the zoomed regions in Fig.4 (a) and (b)). We applied the AS-LNLM method to processthe HDCT images using the parameters given in TABLE I. This parameter setting was set to find the optimal parameter combination that provided the best qualitative results. This qualitative evaluation was carried out in collaboration with three experienced radiologists (refer to Section. IV. B. 2)). Fig.4 (c) and (d) illustrate the corresponding processed HDCT images of Fig.4 (a) and (b) from the AS-LNLM method. We can see in Fig.4 (c)-(d) that the AS-LNLM method leads to more preferable CT images with a better noise/artifact suppression. Thus instead of using the original HDCT images as the ground-truth reference images, we will consider thereafter the processed HDCT images for quantitative calculations in this phantom experiment.

In the implementation of the LNLM and the AS-LNLM method, different parameter settings were used for the visualizations in mediastinum and lung windows. For reminder, the AS-LNLM method involved 7 parameters to set, namely the total decomposition scale $S$ of SWT, the $K$, iter and inc for the nonlinear diffusion, and the decaying parameter $h$ and the sizes of $n$ and $N$ for the subsequent LNLM processing. Practically, in the implementation of the AS-LNLM processing, we set $S$ to 2 because in SWT operation most high frequency features are found concentrated within the first two scales, and the iteration number iter and step size inc in the nonlinear diffusions, and the sizes of $n$ and $N$ are fixed, and $K$ and $h$ are modulated to obtain the visually good results in mediastinal and lung windows. In the following, only $h$ related to the LNLM filter and the $[K, h]$ used in the AS-LNLM method, will be specified and discussed. As for the AS-LNLM method, the parameter setting for the HDCS method was set based on [19] to provide the best qualitative results. We present first a visual assessment of the performance, then a quantitative evaluation based on the signal to noise ratio (SNR) and the standard deviation computation(STD).

\section{1) Visual Assessment:}

Fig.5 (a) and Fig.6 (a) display the original and processed LDCT $16^{\text {th }}$ slice of the volume respectively using the mediastinal and lung windows. We observe that the LDCT image quality is significantly degraded by mottled noise and streak artifacts. Results are provided after the application of the HDCS 
filter (Fig.5 (b) and Fig.6 (b)), LNLM filter with a decaying parameter $h$ respectively equal to 10 (Fig.5 (c) and 6 (c)) and 200 (Fig.5 (d) and 6 (d)), AS-LNLM method (Fig.5 (e) and 6 (e)). The HDCS filter does not completely remove the streak artifacts and introduce some false structures which are not presents in the original LDCT images (see the red dash circles superposed on the images). These aliasing structures were introduced by the 3D processing that takes into account the neighboring slices. A small value of $h$ in the LNLM method does not allow a good suppression of the streak artifacts in LDCT image (see the zoomed ROI in Fig.5 (c) and Fig.6 (c)), and increasing $h$ tends to enforce the smoothing of the structures and make disappear the thin features or on the contrary remove the slight discontinuity between two structures (see the zoomed ROI in Fig.5 (d) and Fig.6 (d)). The AS-LNLM method provides a good compromise between removing the streak artifacts and smoothing the features while preserving the edges. Fig.5 (f) provides an example of the LDCT image (mediastinal window) that is obtained when performing the FBP reconstruction using the smoothing kernel B31f: edges were blurred and artifacts were not effectively suppressed. If now we compare these results with the ground truth images (Fig.4 (c) and (d)), we can conclude that the AS-LNLM method leads to a better structure preservation and noise/artifacts reduction than the HDCS and the LNLM methods.
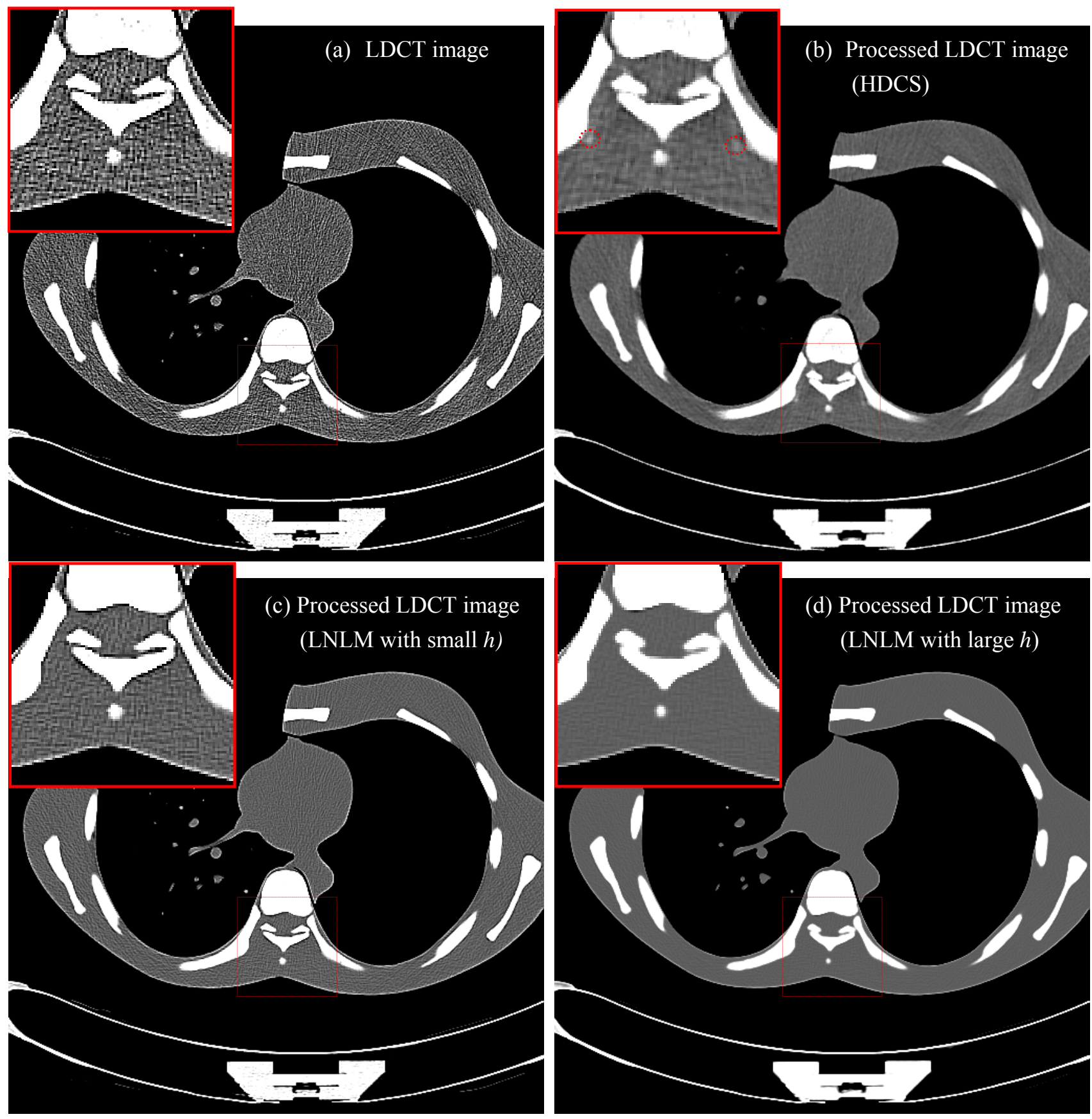

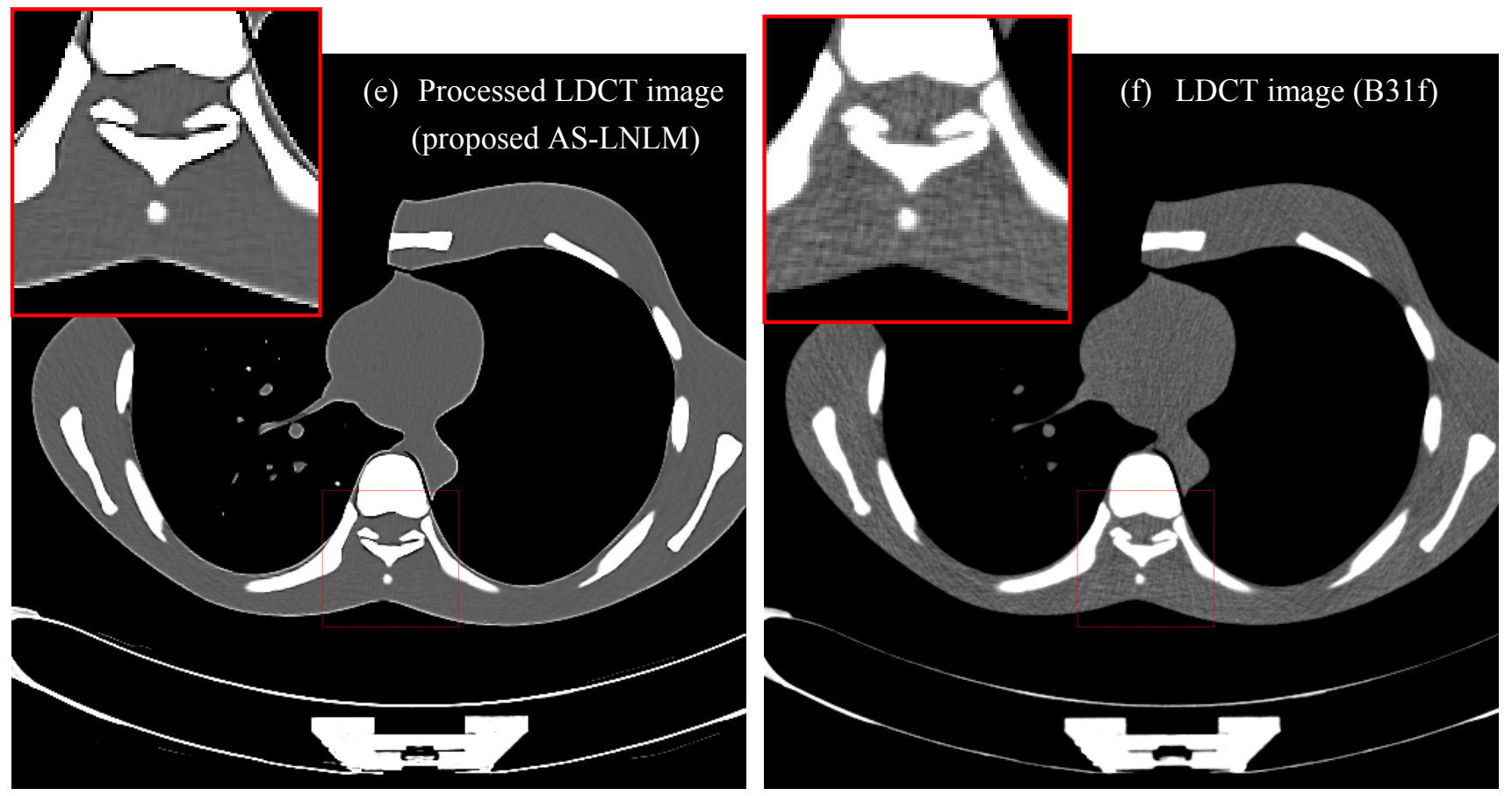

Fig. 5. Visualization of the $16^{\text {th }}$ slice of the volume of both original and processed LDCT thoracic phantom, in a mediastinal window. (a), original LDCT image (30 mAs); (b), LDCT image after HDCS filtering [19]; (c), LDCT image after LNLM filtering with $h=20$; (d), LDCT image after LNLM filtering with $h=200$; (e), LDCT image after application of the AS-LNLM method with $[K=200, h=20]$; (f), original LDCT image reconstructed with a smoothing kernel B31f.
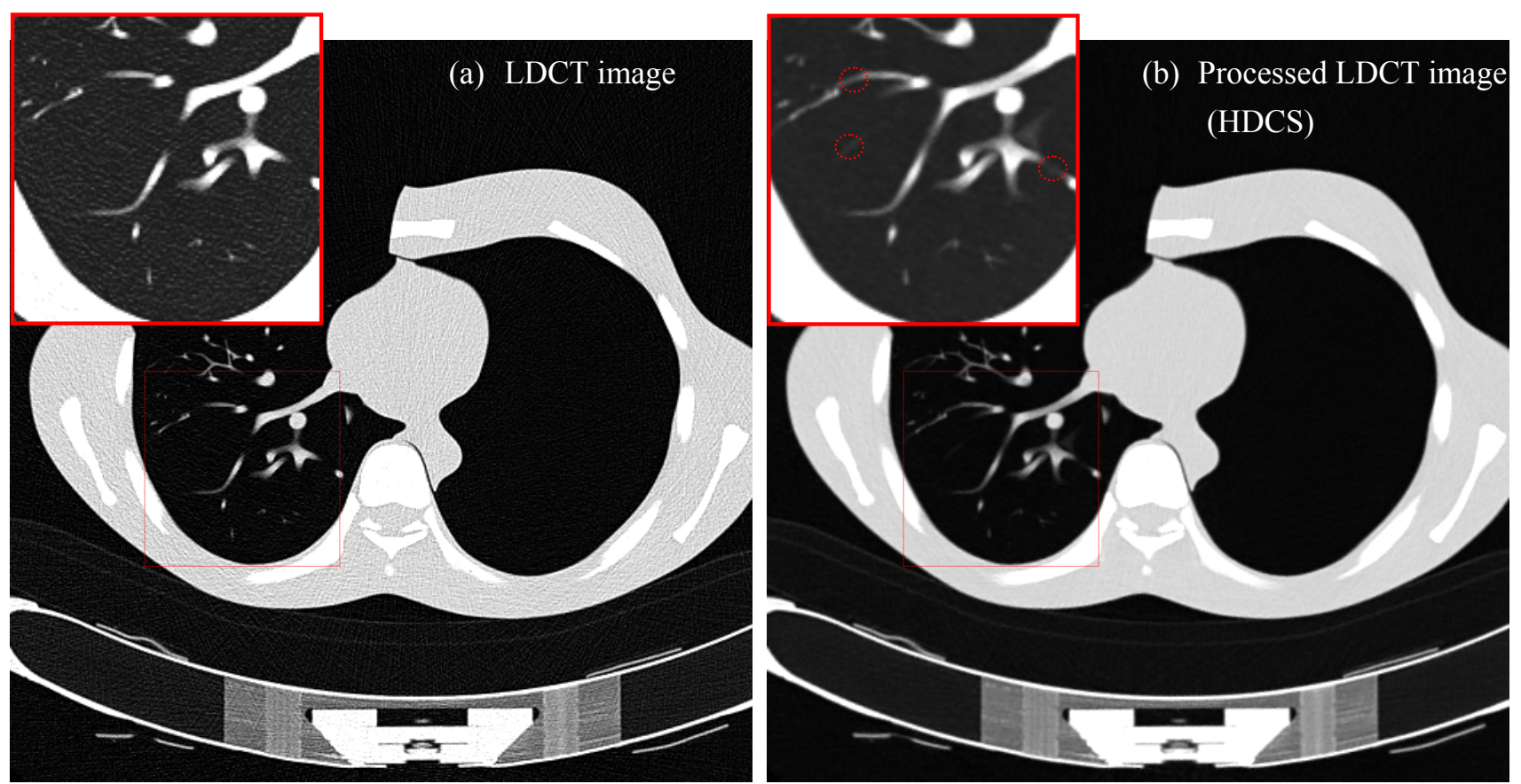

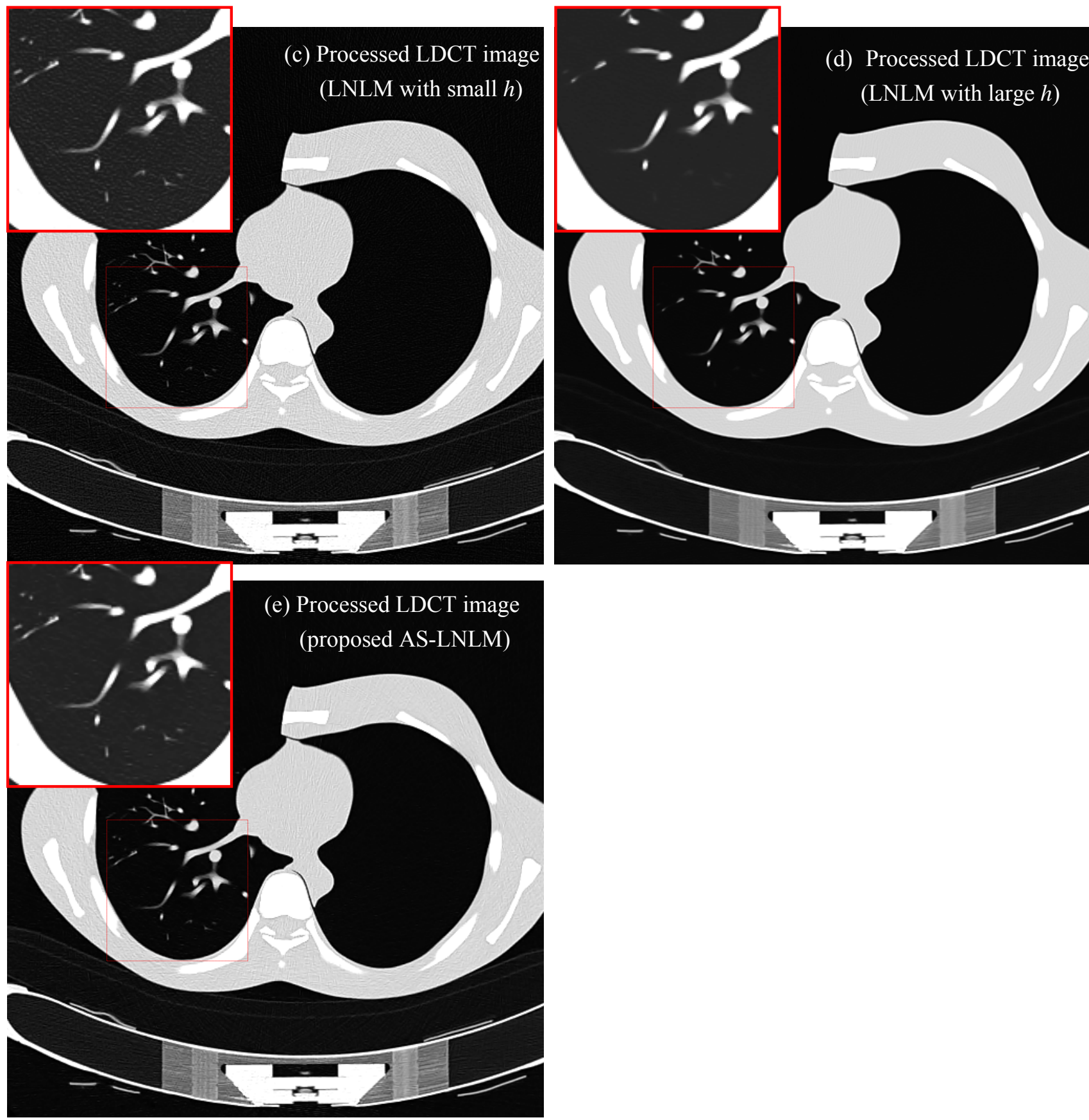

Fig. 6. Visualization of the $16^{\text {th }}$ slice of the volume of both original and processed LDCT thoracic phantom, in a lung window. (a), original LDCT image (30 mAs); (b), LDCT image after HDCS filtering [19]; (c), LDCT image after LNLM filtering with $h=20$; (d), LDCT image after LNLM filtering with $h=200$; (e), LDCT image after application of the AS-LNLM method with $[K=100, h=5]$.

\section{2) Quantitative Assessment:}

We computed the SNR with respect to the ground truth data (processed HDCT images), and then the STD for selected homogeneous regions. This STD is computed for all the original LDCT images and the processed images (from HDCS, LNLM and AS-LNLM methods). We perform analysis in both mediastinum and lung windows. Attenuation intensities in Hounsfield units were used in calculations. The SNR is calculated as follows:

$$
\mathrm{SNR}=\frac{1}{Z} \sum_{z} 10 \log _{10}\left(\sum_{i}\left(F_{i}^{z}-\bar{F}^{z}\right)^{2} / \sum_{i}\left(F_{i}^{z}-\widehat{f}_{i}^{z}\right)^{2}\right)
$$

where, with $Z$ representing the total slice number, $\hat{f}^{z}$ denotes the slice $z$ for test image $\hat{f}$. $F^{z}$ and $\bar{F}^{z}$ define the reference image and the mean intensity of this image respectively. Three $40 \times 40$ homogeneous regions have been selected and for each slice of each volume (original LDCT and 
processed LDCT), the averaged STD is computed:

$$
\begin{aligned}
& \mathrm{STD}=\frac{1}{3}\left(\operatorname{STD}_{\Omega 2}+\operatorname{STD}_{\Omega 2}+\operatorname{STD}_{\Omega 3}\right) \\
& \operatorname{STD}_{\Omega}=\frac{1}{z} \sum_{z}\left(\frac{1}{|\Omega|-1} \sum_{i \in \Omega}\left(\hat{f}_{i}^{z}-\overline{\widehat{f}}_{\Omega}^{z}\right)^{2}\right)^{\frac{1}{2}}
\end{aligned}
$$

where, $\overline{\widehat{f}}_{\Omega}^{z}$ corresponds to the mean intensities of region $\Omega(\Omega 1, \Omega 2$ and $\Omega 3)$ in slice z. $\Omega 1, \Omega 2$ and $\Omega 3$ represent the three selected homogeneous regions depicted in Fig.8, and $|\Omega|$ denotes the pixel numbers of the selected region $\Omega$.
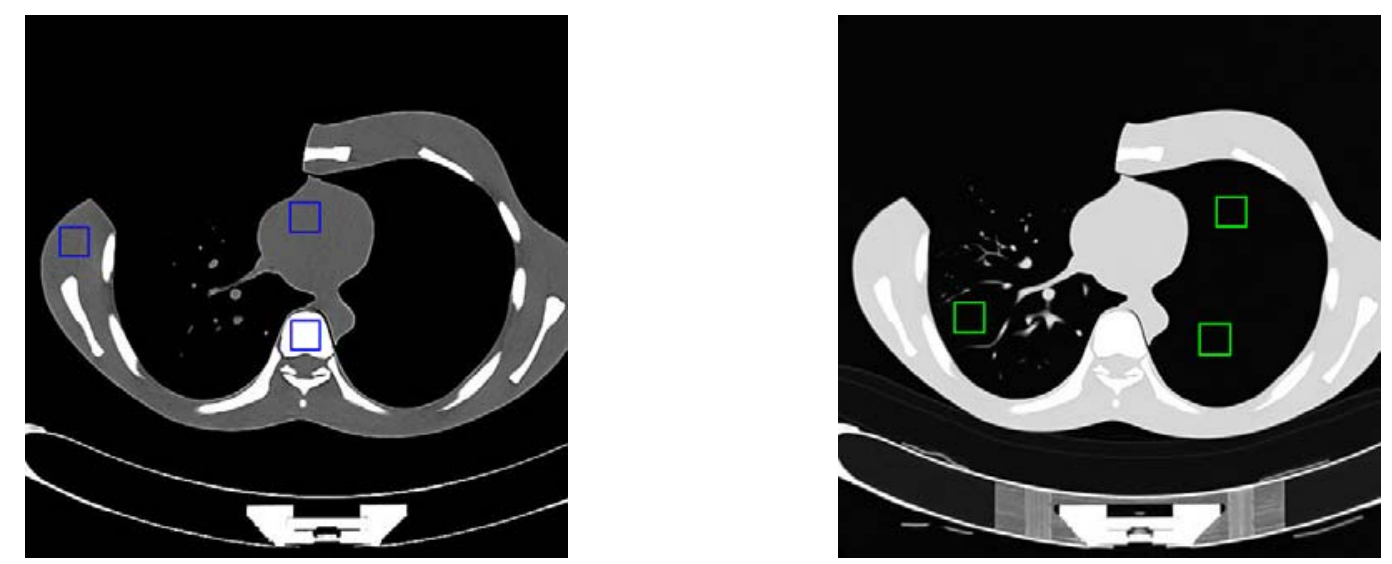

Fig. 7. Selected homogeneous regions $(\Omega 1, \Omega 2$ and $\Omega 3)$ in mediastinum window (left) and lung window (right) for STD calculation.

TABLE II. THE CALCULATED SNR AND STD IN THE PHANTOM EXPERIMENT

\begin{tabular}{|c|c|c|c|c|}
\hline \multirow{2}{*}{ Images } & \multicolumn{3}{|c|}{ Mediastinum window } & \multicolumn{2}{c|}{ Lung window } \\
\cline { 2 - 5 } & SNR & STD & SNR & STD \\
\hline Original LDCT images & 19.96 & 75.20 & 19.96 & 29.97 \\
\hline Processed LDCT from HDCS & 22.92 & 39.29 & 22.92 & 7.01 \\
\hline Processed LDCT images from LNLM $($ small $h)$ & 22.81 & 35.44 & 20.95 & 21.95 \\
\hline Processed LDCT images from LNLM $($ large $h)$ & 20.95 & 9.55 & 20.37 & 3.56 \\
\hline Processed LDCT images From AS-LNLM & $\mathbf{2 4 . 6 3}$ & $\mathbf{2 3 . 6 1}$ & $\mathbf{2 4 . 4 2}$ & $\mathbf{2 . 6 2}$ \\
\hline Original LDCT images with smoothing kernel B31f & 20.95 & 17.58 & & \\
\hline Reference Images (Processed HDCT images From AS-LNLM) & & 21.64 & & 2.25 \\
\hline
\end{tabular}

TABLE II provides the calculated comparative SNR and STD in the both two cases of mediastinum and lung windows. It allows the concluding on the supremacy of the AS-LNLM method that brings the highest SNR, and the STD closest to that of the reference images. TABLE II also points out that, for the LNLM method, a large value of $h$ lowers the STD in the homogenous regions but it also leads to a lower SNR.

Computation costs are given in Table III for the HDCS method, the CUDA accelerated LNLM method and the AS-LNLM methods. The total time (in CPU seconds) is given for the processing of a $512 \times 512 \times 40$ CT dataset. The HDCS filter computation time is low comparing with the other methods. The optimized parallelization for the AS-LNLM method allows speeding up this computation time by more than 100 times, when comparing with its serial version. 
TABLE III. COMPARISON OF CPU TIME COST (SECOND) BETWEEN THE DIFFERENT METHODS

\begin{tabular}{|c|c|}
\hline Different methods & CPU time cost (seconds) \\
\hline HDCS method & 75 \\
\hline LNLM method (CUDA accelerated) & 407 \\
\hline AS-LNLM method (CUDA accelerated) & 384 \\
\hline AS-LNLM method (CPU serial version) & 43145 \\
\hline
\end{tabular}

\section{B. Patient Data Experiment}

\section{1) Visual Assessment:}

The anthropomorphic phantom and human chest data suffer at quite similar degrees from noise/artifacts when the acquisition is performed in the same low dose scan protocol. So here the parameter settings selected by experts in phantom experiments (refer to TABLE I) were re-used for patient data. This strategy of parameter setting has been validated by the experts.

Fig.8 and Fig.9 show the original and processed LDCT images (30mAs) of a 59 years old man in different windows. Fig.8 (e)-(h) and Fig.9 (d)-(f) are the selected zoomed regions. Mottle noise and streak artifacts can be easily observed in the original LDCT images depicted in Fig.8 (a) and Fig.9 (a). Results provided by the HDCS are displayed in Fig.8 (b) and Fig.9 (b). As previously observed on the thoracic phantom, the same false structures appears that relate to the introduction of some aliasing features (See in Fig.8 (f) and Fig.9 (e) the structures in red dash circles). The AS-LNLM method has been applied with two different parameter settings $[K=200, h=20]$ and $[K=100, h=5]$ for mediastinum and lung windows, and the results were given in Fig.8 (c) and Fig.9 (c). The LDCT image reconstructed using the built-in smoothing kernel B31f (Fig.8 (d)) is currently clinically used for mediastinum window in Siemens CT system, and we can find the overall image contrast was degraded when using this smoothing kernel. Compared to other images, the AS-LNLM method can lead to images with a better structure preservation (see the ascending aorta walls pointed by red arrows in Fig.8 (e)-(h)) and noise /artifact reduction in both mediastinum and lung windows.

In the supplementary file affiliated to this paper, two other patient examples are shown in Fig. 10 and Fig.11 (two men of 62 and 68 years old respectively) The supplementary file also includes a visual comparison (Fig. 12 and Fig. 13) between the proposed method and the Adaptive Statistical Iterative Reconstruction (ASIR) algorithm developed in the latest GE CT750 HD system [34]. The results in Fig.10-Fig.13 also validated the above merits of the proposed processing. 
(a) LDCT image (B70f)

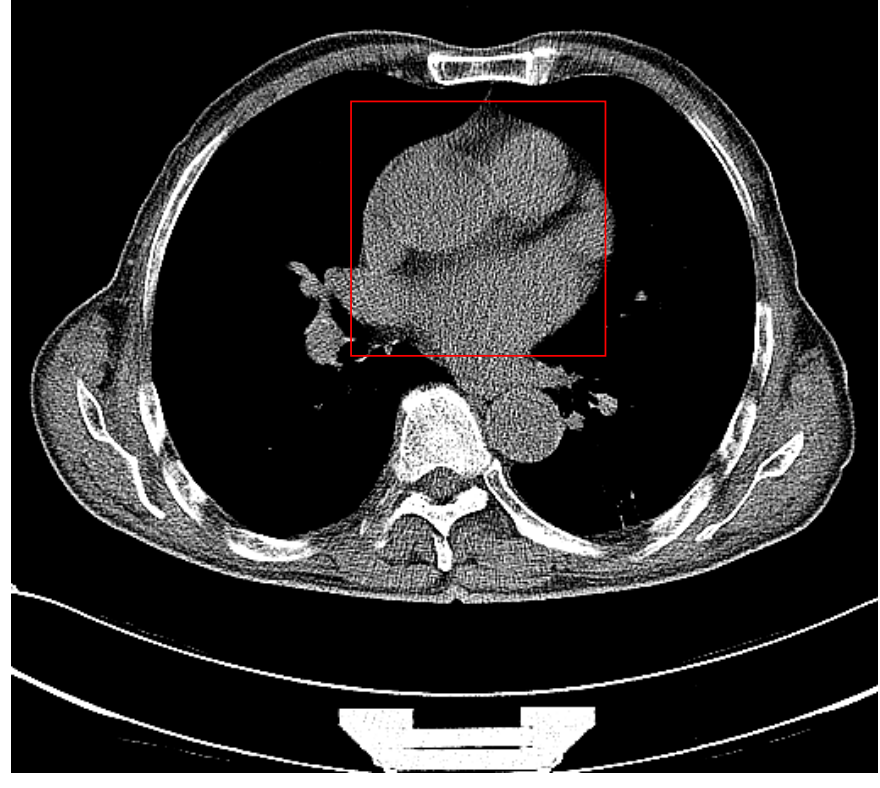

(c) Processed LDCT image (proposed AS-LNLM)
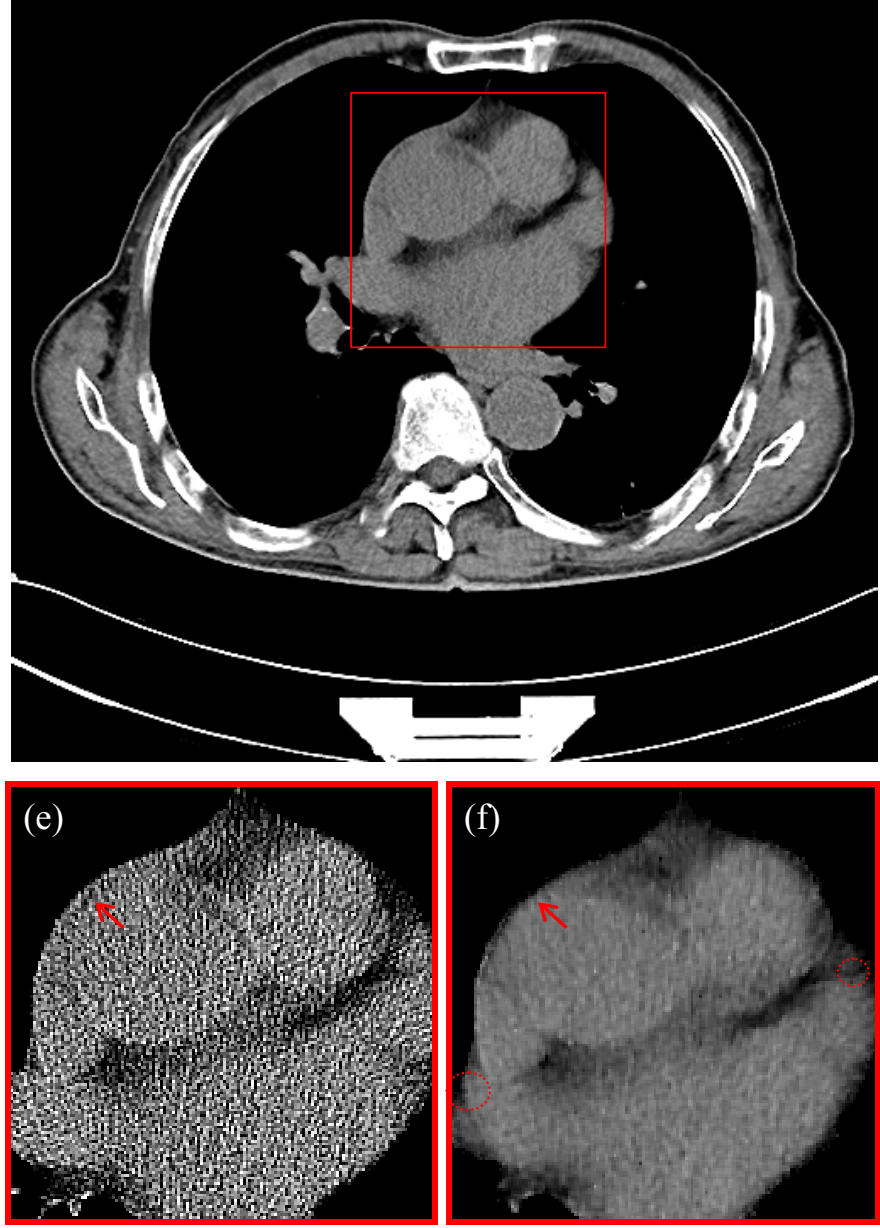

(b) Processed LDCT image (HDCS)

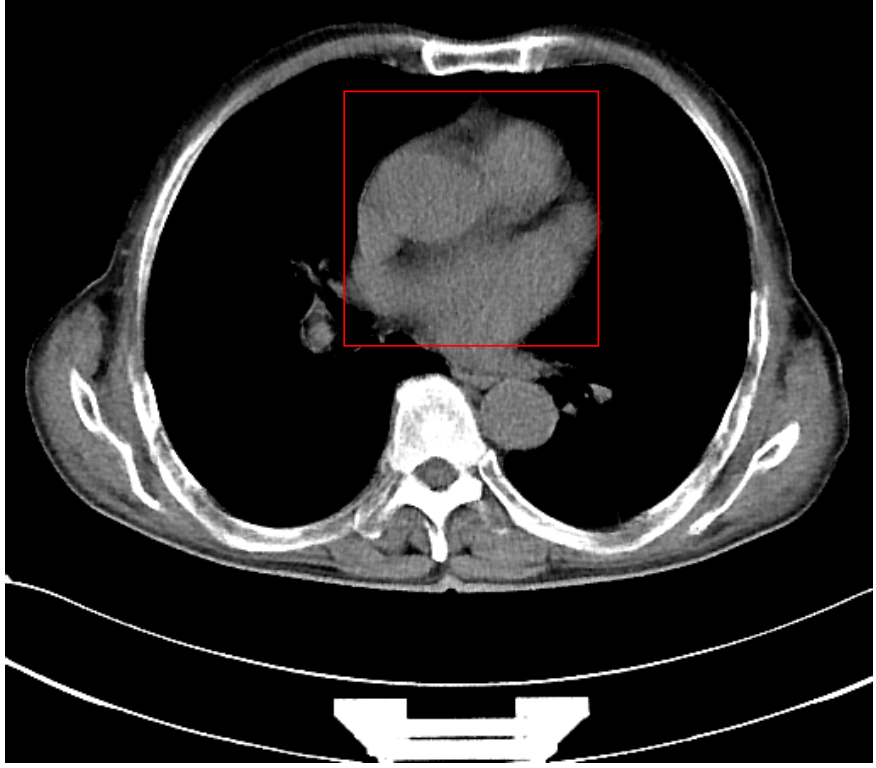

(d) LDCT image (B31f)
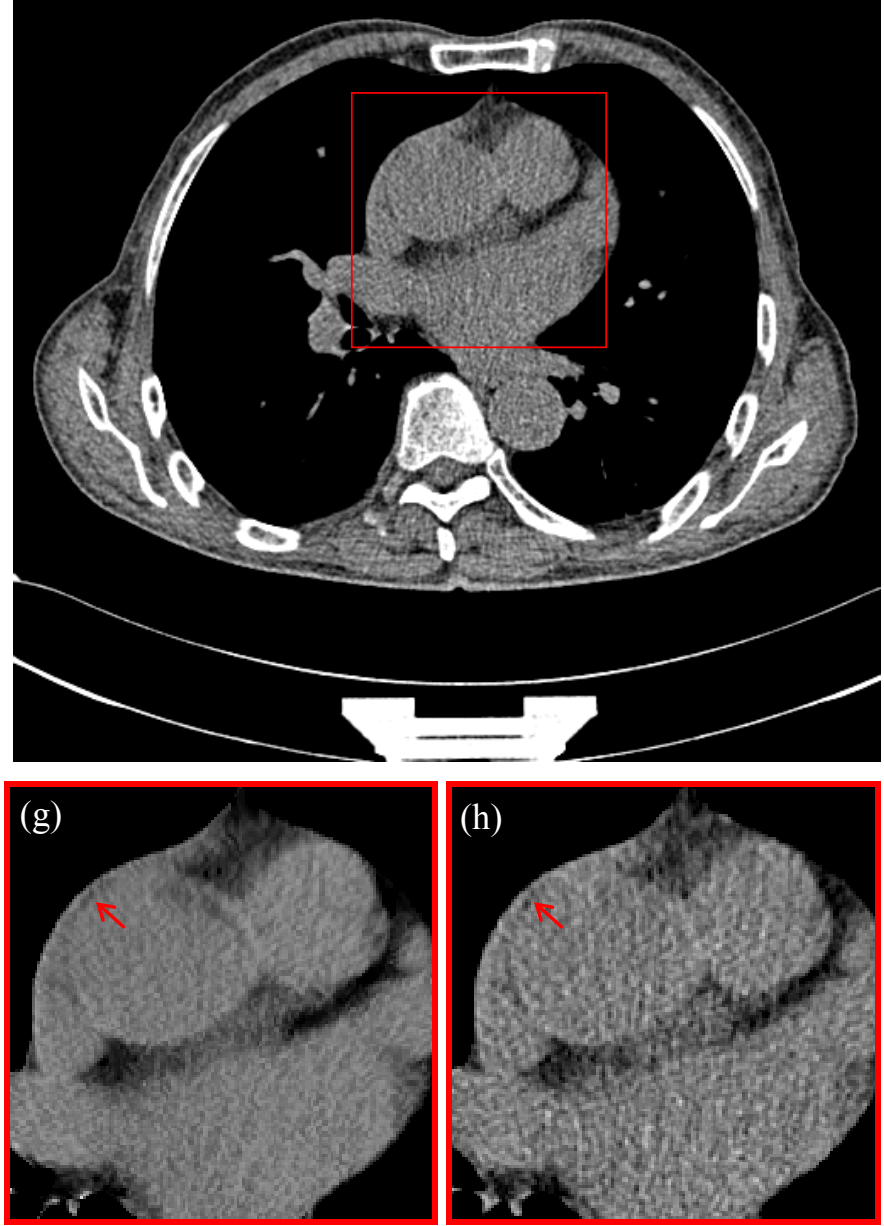

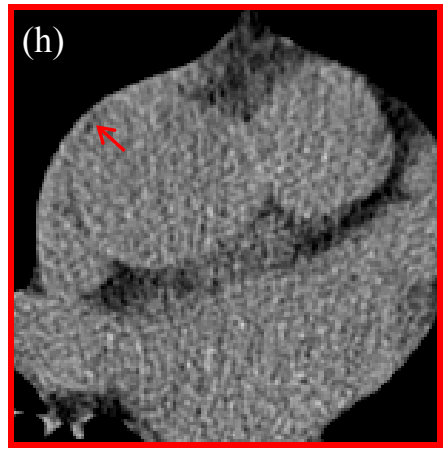

Fig. 8. Visualization of the original and processed LDCT images (30mAs) of a 59 years old man using a mediastinal window. (a), original LDCT image reconstructed with the kernel B70f; (b), LDCT images after HDCS filtering; (c), LDCT image after application of the AS-LNLM method; (d), original LDCT image reconstructed with the kernel B31f; (e)-(h) shows the zoomed ROI in (a)-(d). 

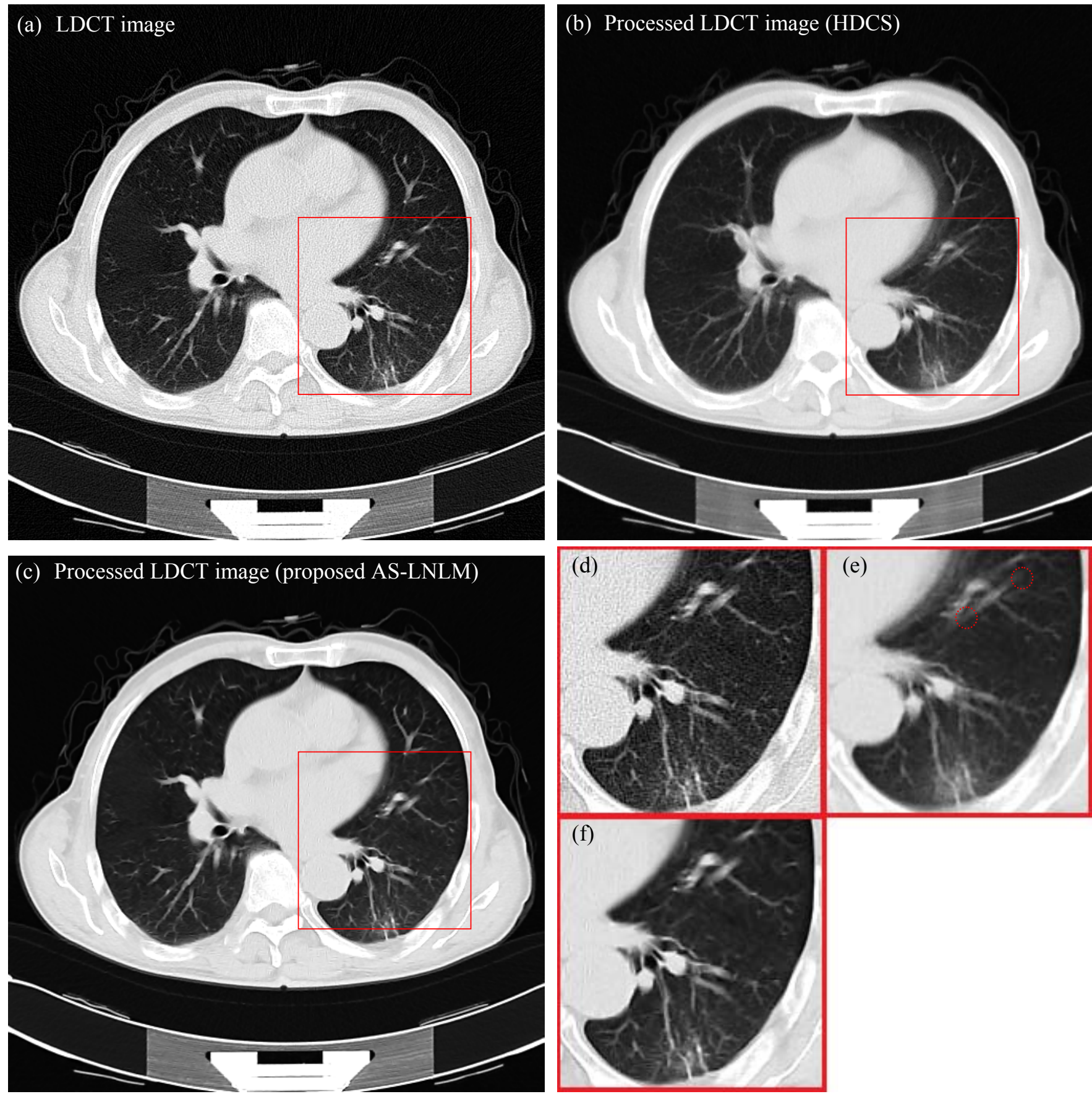

Fig. 9. Visualization of the original and processed LDCT images (30mAs; reconstruction kernel B70f) of a 59 years old man using a lung window. (a), original LDCT image; (b), LDCT images after HDCS filtering; (c), LDCT image after application of the AS-LNLM method; (d)-(f) shows the zoomed ROI in (a)-(c).

\section{2) Qualitative Assessment:}

25 original and 25 AS-LNLM processed LDCT images from the 25 patients were assessed separately for noise suppression, artifact suppression, contrast preservation and overall image quality using a five-point subjective scale (1=unacceptable, 2=substandard, 3=acceptable, 4=above average, $5=$ excellent). Here, we define artifacts as the shapes with a passive effect on subjective diagnosis. Three radiological readers (R1 (X.D.Y. with 15 years of experience.), R2 (X.H.Y. with 8 years of experience.), R3 (Y.M.D. with 5 years of experience.) independently evaluated the randomized LDCT images, HDCT images and the AS-LNLM processed LDCT images in a digital DICOM archiving/assessing workstation (ViewDEX 2.0 [35]). To compare the processed LDCT images with routine HDCT images, we selected 25 thoracic HDCT images (120mAs) of other patients from previous scans in the database. All the involved patients for these HDCT images gave their written consent to use 
their CT images. In this way, the 4 image features were assessed in all the 75 images, which include 50 original/processed LDCT images, and 25 HDCT images. This results in a total of 900 parameter ratings for the 4 image quality parameters and the 3 readers $(75 \times 4 \times 3=900)$. For each subset of images, the 4 image scores were reported as means \pm SDs (averaged scores of the 3 radiologists \pm standard deviations). The subjective quality parameters of the original LDCT images and the processed LDCT images were compared with those of the original HDCT images. Individual subjective image quality scores for each parameter were compared using the Wilcoxon signed rank test (SAS/STAT software; SAS Institute, Cary, NC). The differences between each two groups were determined by the Student $t$ test (Excel; Microsoft) with $P<0.05$ considered a statistically significant difference. As illustrated in TABLE IV, all the 3 readers rated that, for all the 4 image quality scores, the qualities of the original LDCT images were inferior to those of the processed LDCT images and the original HDCT images. In TABLE IV, statistically significant differences between the original HDCT and LDCT images $(P<0.05)$ are noted in all the subjective image quality scores. And with reference to the original HDCT images, there are no statistically significant differences of the 4 subjective quality scores for the processed LDCT images $(P>0.05)$. TABLE IV also shows that the AS-LNLM processing leads to CT images with qualities even higher than the original HDCT images.

TABLE IV. IMAGE QUALITY SCORES ( mean \pm SDs ) FOR THE ORIGINAL AND AS-LNLM PROCESSED LDCT IMAGES, AND THE ORIGINAL HDCT IMAGES

\begin{tabular}{|cccccc|}
\hline Image Denoising & $\begin{array}{c}\text { Tube Current } \\
\text { settings (mAs) }\end{array}$ & $\begin{array}{c}\text { Noise } \\
\text { Suppression }\end{array}$ & $\begin{array}{c}\text { Artifact } \\
\text { Suppression }\end{array}$ & $\begin{array}{c}\text { Contrast } \\
\text { Preservation }\end{array}$ & $\begin{array}{c}\text { Overall } \\
\text { Image Quality }\end{array}$ \\
\hline None & $30 \mathrm{mAs}$ & $1.52 \pm 0.34 *$ & $1.77 \pm 0.41 *$ & $1.87 \pm 0.38^{*}$ & $1.90 \pm 0.43 *$ \\
\hline None & $120 \mathrm{mAs}$ & $3.21 \pm 0.32$ & $3.36 \pm 0.25$ & $3.46 \pm 0.32$ & $3.30 \pm 0.29$ \\
\hline Processed & $30 \mathrm{mAs}$ & $3.53 \pm 0.31$ & $3.53 \pm 0.32$ & $3.52 \pm 0.29$ & $3.47 \pm 0.36$ \\
\hline \multirow{4}{*}{$*$ Significantly $(P<0.05)$} & different from the mean scores for HDCT images. \\
\hline
\end{tabular}

Inter-observer agreements on the above 4 quality scores were accessed by the Bland-Altman statistic method [36] . For the cases without known truth, the Bland-Altman method assumes $95 \%$ of variations lie between the mean difference plus or minus $2 \times \mathrm{SD}$ (Standard Deviation) of the variations. These are termed the $95 \%$ limits of the agreements that represent the limits within which the true values will lie. Fig. 14 (1)-(3) display the Bland-Altman plots for the 4 quality scores for the scoring agreements between R1 and R2, R1 and R3, R2 and R3, respectively. We can see in Fig. 14 that, for the 4 image quality scores, the $95 \%$ limits between the 3 readers lie within the range from -0.4 to 0.4 , which implies a substantial overall concordance among the scores of the 3 readers.
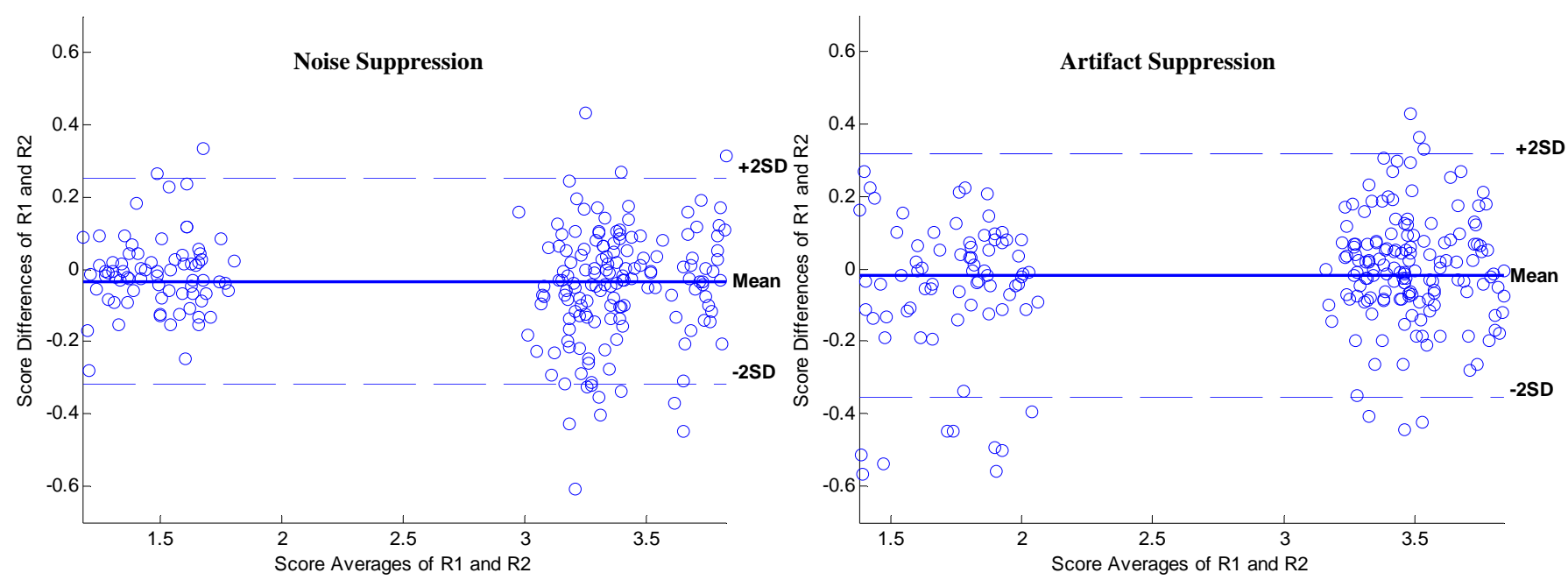

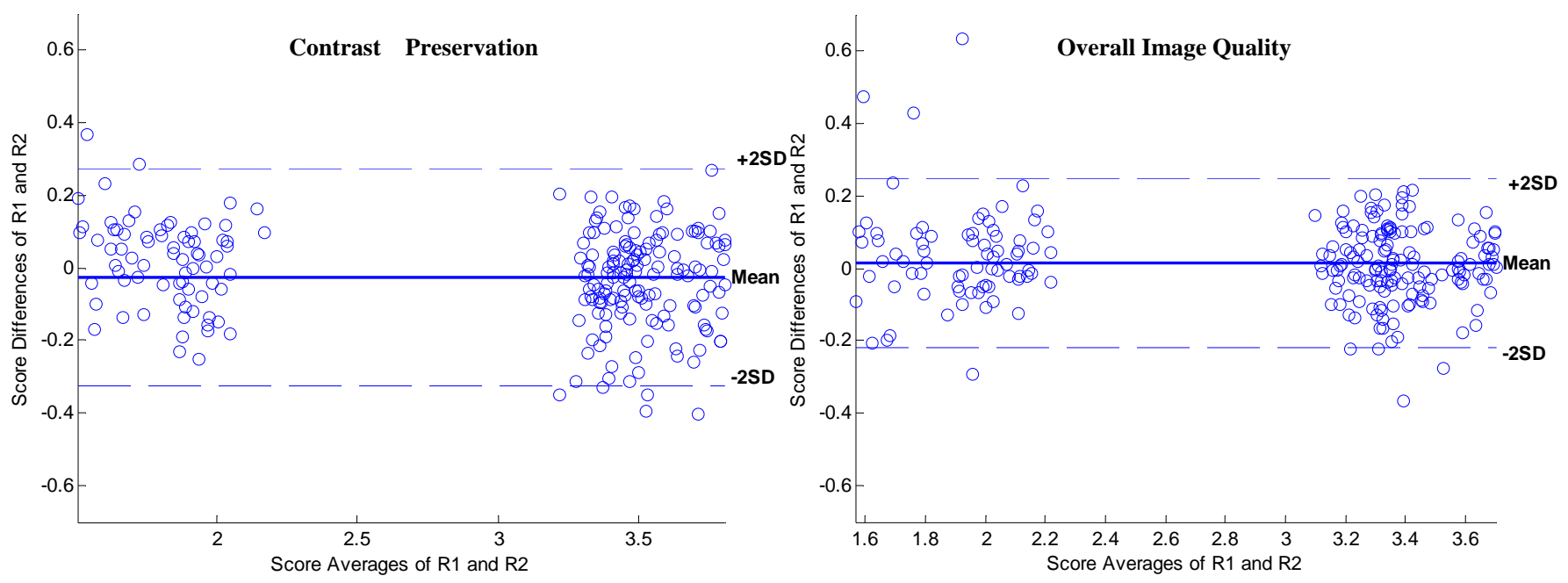

(1) Bland and Altman plot of interobserver agreement between R1 and R2
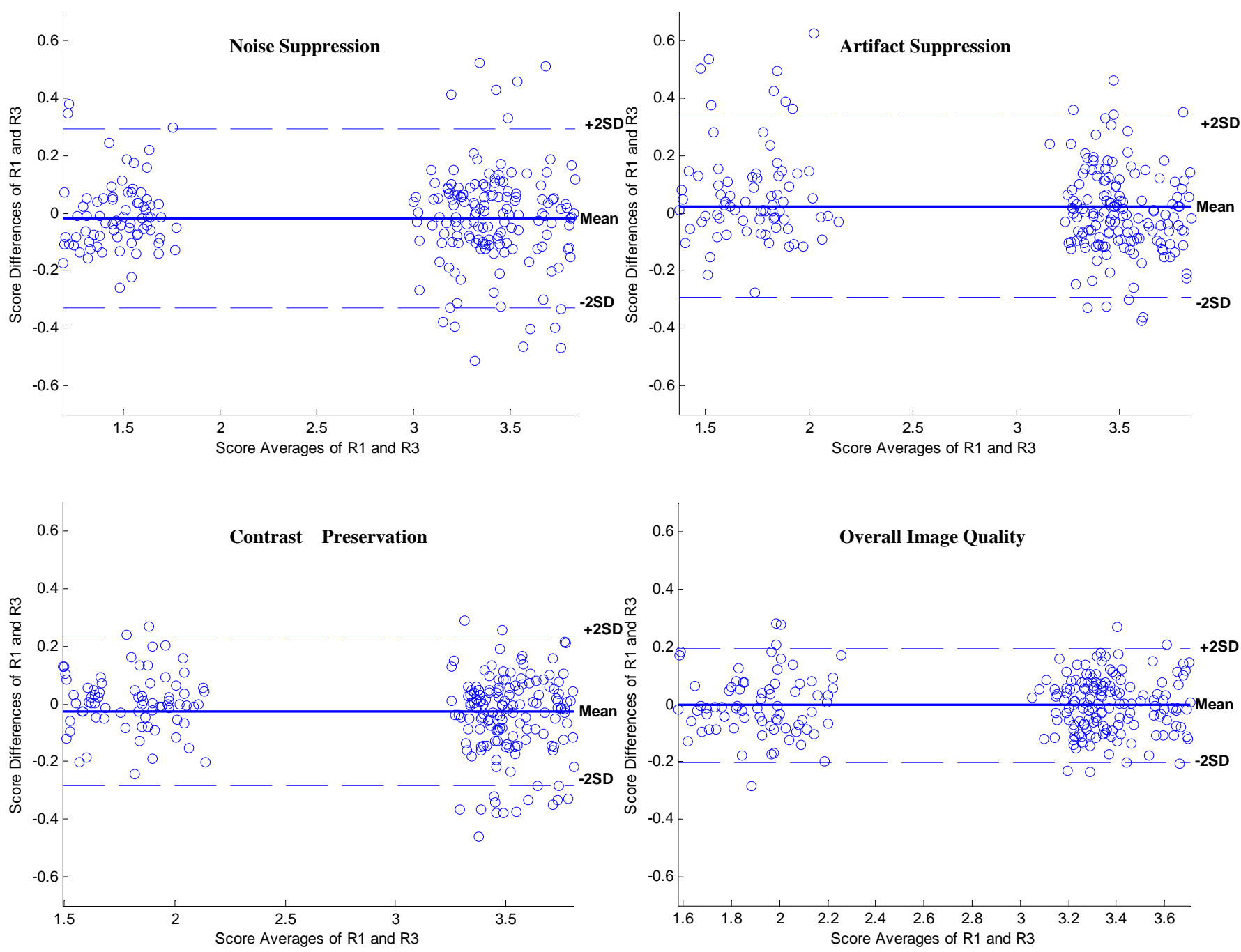

(2) Bland and Altman plot of inter-observer agreement between R1 and R3 

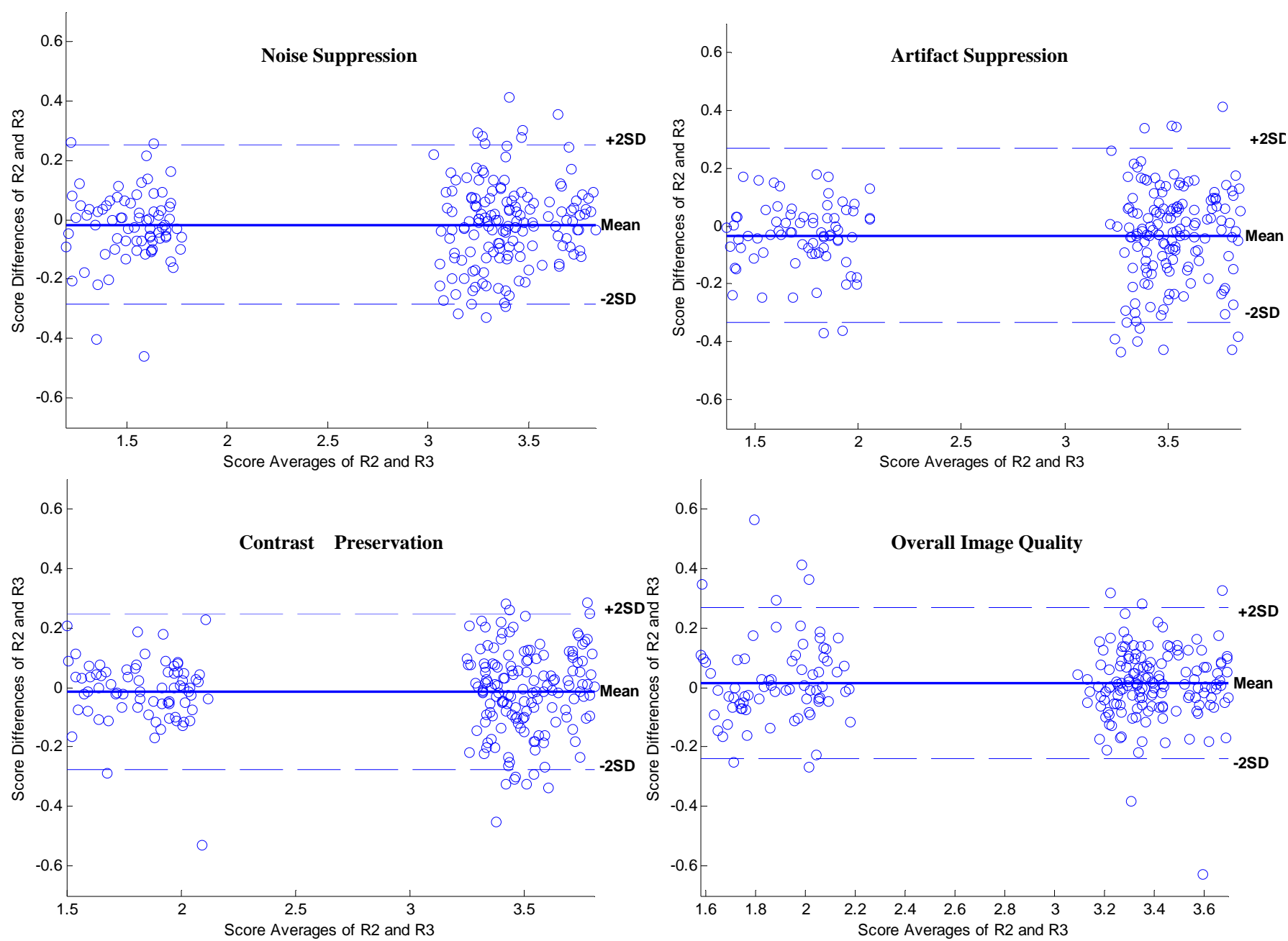

(3) Bland and Altman plot of inter-observer agreement between R2 and R3

Fig. 10. Bland and Altman analysis of inter-observer agreements.

\section{Discussion}

Visual results in Fig.5-Fig.6 and Fig.8-Fig.11 show that the proposed AS-LNLM processing behaves better than the HDCS and LNLM methods in the suppression of noise/artifacts. Notably, in Fig.5-Fig.6, Fig.8-Fig.9 and S.1-S.2 (in the supplementary file) we can see the HDCS processing tends to introduce some false structures into the processed images. Fig.5 and Fig.6 illustrate that suppressing the streak artifacts by using large decaying parameter will lead to blurred anatomical structures in the LNLM processing. Especially in the supplementary file, S.3 and S.4 show that the proposed processing can potentially achieve results with comparable performance as the iterative reconstruction used in the latest GE CT system. To evaluate the proposed method quantitatively, TABLE II lists the SNR with respect to the reference phantom images (in Fig.4 (c)-(d)) and the STD for selected homogenous regions. TABLE II validates that the proposed processing can lead to processed thoracic LDCT images with the highest SNR and the closest STD with respect to the reference images. In qualitative test, as to the 4 subjective image scores (noise suppression, artifact suppression, contrast preservation and overall image quality), notable improvements over the original LDCT images were obtained $(P<0.05)$ for the AS-LNLM processed LDCT images. The Bland and Altman analysis in Fig.10 also shows there is substantial overall concordance among the scores of the 3 readers.

Although this algorithm demonstrated a good potential in improving thoracic LDCT images, the following limits should also be noted: In S.1 (f) and S.2 (f), we can still discern some artifacts remaining 
in the images processed by the AS-LNLM method, and some tiny structures tend to be obscured when some aggressive parameters were used to suppress the artifacts and noise. The reason is that, for the LDCT images scanned from human breast parts with much high-density tissues, some streak artifacts are too severe to be well suppressed. In this work the parameters calibrated in the phantom test were reused in routine clinical experiments. This strategy of parameter setting is based on the assumption that the anthropomorphic thoracic phantom and human chests suffer from quite similar noise/artifacts disturbance when using the same scan protocols in the same CT scanners. Though proved effective by above experiments, the parameter settings trained in phantom experiments might not produce satisfying results for some patients with abnormal body shapes, so empirical modulation may be needed in those cases. Also, TABLE III shows that nearly 10 seconds are needed for processing one $512 \times 512$ DICOM image even after GPU acceleration. This might form a computational burden for those radiological units that require fast and real-time clinical diagnosis.

\section{Conclusion}

This paper presented a two-step method named AS-LNLM to improve the quality of thoracic LDCT images. In this AS-LNLM method, before the operation of LNLM, the streak artifacts in LDCT images were suppressed by directional 1-D nonlinear diffusions in stationary wavelet domain. Compared to ordinary processing methods in single image scale, artifact-suppressing operations on high frequency wavelet subbands can produce less obscuring on the original low frequency information, which suffers less from artifacts and noise. The parameter settings in processing the patient data can be tractably made according to the previous phantom experiment results validated by radiological expert. In addition, to enhance clinical applicability, a parallel acceleration using GPU-based CUDA has been made.

A CT workstation is currently under development to facilitate the on-going clinical evaluations and applications. LDCT images with smaller nodules will be used to further test the proposed AS-LNLM method. We are also trying extending the method from 2D to 3D with the objective to further suppress the streak artifacts by taking into account the 3D local geometries. An improved nonlinear diffusion, with parameters controlled by the artifact directional prominence, is also being devised and tested. Other work in the future will include further lowering the computation cost by testing the processing under more efficient hardware architectures, exploring the applications with thinner slice thicknesses $(<2 \mathrm{~mm})$, and taking a thorough comparison between the proposed processing with iterative reconstruction algorithms.

\section{Acknowledgment}

This research was supported by National Basic Research Program of China under grant (2010CB732503), National Natural Science Foundation under grants $(81000636,31100713,81101104,60801009)$, and the Project supported by Natural Science Foundations of Jiangsu Province (BK2009012 and BK2011593). The authors also thank the Kyoto Kagaku Company for providing the phantom "n1 Lungman". We also show great appreciation for the constructive comments of the reviewers.

\section{References}

[1] American Cancer Society, Cancer Facts and Figures. (2010).

[2] MacRedmond R, Logan P. M, Lee M, et al (2004) Screening for lung cancer using low dose CT scanning. Thorax 59:237-41.

[3] MacRedmond R, McVey G, Lee M, et al (2006) Screening for lung cancer using low dose CT scanning: results of 2 year follow up. Thorax. 61: 54-56.

[4] Brenner D.J and Hall E.J (2007) Computed tomography-an increasing source of radiation exposure. New England Journal of Medicine 357: 2277-2284.

[5] Mannudeep K and Michael M.M, et al (2004) Strategies for CT Radiation Dose Optimization. Radiology 230: 
619-628.

[6] Angel E, Yaghmai N, Jude C.M, et al (2009) Monte Carlo simulations to assess the effects of tube current modulation on breast dose for multidetector CT. Physics in Medicine and Biology 54(3): 497-512.

[7] Nelson R (2009) Low-Dose CT Screening for Lung Cancer Produces High Rate of False Positives, American Society of Clinical Oncology (ASCO) 45th Annual Meeting: Abstract CRA1502.

[8] Hsieh J (1998) Adaptive streak artifact reduction in computed tomography resulting from excessive x-ray photon noise. Medical Physics 25:2139-2147.

[9] Wang J, Lu H.B, Wen J.H and Liang Z.R (2008) Multiscale penalized weighted least-squares sinogram restoration for low-dose X-ray computed tomography. IEEE Transactions on Biomedical Engineering 55 (3): $1022-1031$.

[10] Kubo T, Ohno Y, Gautam S, Lin P.J, Kauczor H.U, Hatabu H (2008) Use of 3D adaptive raw-data filter in CT of the lung: effect on radiation dose reduction. American Journal of Roentgenology 191(4):1071.

[11] Chen Y, Gao D. Z, Nie C, Luo L.M, Chen W.F, et al (2009) Bayesian statistical reconstruction for low-dose x-ray computed tomography using an adaptive-weighting nonlocal prior. Computerized Medical Imaging and Graphics 33: 495-500.

[12] Zbijewski W, Defrise M, Viergever M, and Beekman F (2007) Statistical reconstruction for x-ray CT systems with non-continuous detectors. Physics in Medicine and Biology, 52(10): 403-418.

[13] Borsdorf A, Raupach R, Flohr T, Hornegger J (2008) Wavelet based noise reduction in CT-images using correlation analysis. IEEE Transactions on Medical Imaging 27:1685-703.

[14] Kalra M.K, Wittram C, Maher M.M, et al (2003) Can noise reduction filters improve low-radiation-dose chest CT images? pilot study. Radiology 228:257-264.

[15] Martinsen A, Saether H, Olesen D, Wolff P, Skaane P (2010) Improved image quality of low-dose thoracic CT examinations with a new postprocessing software. Journal of Applied Clinical Medical Physics 11(3):32-42.

[16] Paul N.S, Blobel J, Prezelj E, Burey P, et al (2010) The reduction of image noise and streak artifact in the thoracic inlet during low dose and ultralow dose thoracic CT. Physics in Medicine and Biology 55: 1363-1380.

[17] Schilham A.M, van Ginneken B, Gietema H, Prokop M (2006) Local noise weighted filtering for emphysema scoring of low-dose CT images. IEEE Transactions on Medical Imaging 25:451-463.

[18] Schaap M, Schilham A, Zuiderveld K.J, et al (2008) Fast Noise Reduction in Computed Tomography for Improved 3-D Visualization. IEEE Transactions on Medical Imaging 27:1120-1129.

[19] Mendrik A.M., Vonken E.P.A., Rutten A, Viergever M.A, et al (2009) Noise reduction in computed tomography scans using 3D anisotropic hybrid diffusion with continuous switch. IEEE Transactions on Medical Imaging, 28:1585-1594.

[20] Chen Y, Chen W.F, Yin X.D et al (2011) Improving Low-dose X-ray CT Images by Weighted Intensity Averaging over Large-scale Neighborhood. European Journal of Radiology, 80 (2): e42-e49.

[21] Buades A, Coll B, and Morel J.M 2005. A non-local algorithm for image denoising. In Proc. IEEE Computer Vision and Pattern Recognition: 60-65.

[22] Mahmoudi M, and Sapiro G (2005) Fast image and video denoising via non-local means of similar neighborhoods. IEEE Signal Processing Letters 12:839-842.

[23] Coupé P, Yger P, Prima S, Hellier P, Kervrann C, Barillot C (2008) An Optimized Blockwise NonLocal Means Denoising Filter for 3DMagnetic Resonance Images. IEEE Transactions on Medical Imaging 27:425-441.

[24] NVIDIA $\quad$ CUDA $_{T M}$ Programming Guide (Version 3.0), http://developer.download.nvidia.com/compute/cuda/3.0/toolkit/docs/NVIDIA CUDA Programming Guide.pdf

[25] Accelerating MATLAB with CUDA Using MEX Files (White Paper), http://developer.nvidia.com/object/matlab cuda.html

[26] Bart Goossens, Hiep Luong, Jan Aelterman, Aleksandra Pizurica, and Wilfried Philips (2010) A GPU-accelerated real-time NLMeans algorithm for denoising color video sequences," in Proceedings of the 12th International Conference on Advanced Concepts for Intelligent Vision Systems, (ACIVS), vol. 6475 of Lecture Notes in Computer Science: 46-57.

[27] Chen Y, Chen W.F, Luo L.M et al (2011) Joint-MAP Tomographic Reconstruction with Patch Similarity Based Mixture Prior Model. SIAM Multiscale Modeling and Simulation, 9:1399-1419.

[28] Gyaourova A, Kamath C, and Fodor I. K (2002) Undecimated wavelet transforms for image de-noising. Technical report, Lawrence Livermore National Laboratory, UCRL-ID-150931.

[29] Mark J.S (1992) The Discrete Wavelet Transform: Wedding the A Trous and Mallat Algorithms. IEEE Transaction on Signal Processing 40(10): 2464 - 2482.

[30] Cohen A, DeVore R, Petrushev P, Xu H (1999) Nonlinear approximation and the space by $\left(r^{2}\right)$. Am J Math, 121: 587-628.

[31] Lohmann G, Bohn S, Müller K, Trampel R, Turner R (2010) Image restoration and spatial resolution in 7 Tesla magnetic resonance imaging, Magnetic Resonance in Medicine 64(1) 15-22.

[32] Perona P and Malik. J (1990) Scale-space and edge detection using nonlinear diffusion. IEEE Transactions on Pattern Analysis and Machine Intelligence 12(7): 629-639.

[33] Bruzzi J.F, Rémy-Jardin M, Delhaye D, Teisseire A, Khalil C, Rémy J (2006). Multi-detector row CT of 
hemoptysis. Radiographics 26:3-22.

[34] Yanagawa M, Honda O, Kikuyama A, Gyobu T, et al (2011) Pulmonary nodules: Effect of adaptive statistical iterative reconstruction (ASIR) technique on performance of a computer-aided detection (CAD) system-Comparison of performance between different-dose CT scans. European Journal of Radiology, Available online 5 October 2011, ISSN 0720-048X, 10.1016/j.ejrad.2011.09.011.

[35] Hakansson M, Svensson S, Zachrisson S, Svalkvist A, Bath M, Mansson L. G. ViewDEX 2.0 (2009): a Java-based DICOM-compatible software for observer performance studies. Proc SPIE 2009, 7263: 72631G1-72631G10.

[36] Bland J.M. and Altman D.G (1986) Statistical methods for assessing agreement between two methods of clinical measurement. Lancet, i:307-310. 